\title{
Pricing of Fluctuations in Electricity Markets
}

\author{
John N. Tsitsiklis and Yunjian Xu \\ Laboratory or Information and Decision Systems, MIT, Cambridge, MA, 02139, \{jnt@mit.edu, yunjian@mit.edu\}
}

\begin{abstract}
In an electric power system, demand fluctuations may result in significant ancillary cost to suppliers. Furthermore, in the near future, deep penetration of volatile renewable electricity generation is expected to exacerbate the variability of demand on conventional thermal generating units. We address this issue by explicitly modeling the ancillary cost associated with demand variability. We argue that a time-varying price equal to the suppliers' instantaneous marginal cost may not achieve social optimality, and that consumer demand fluctuations should be properly priced. We propose a dynamic pricing mechanism that explicitly encourages consumers to adapt their consumption so as to offset the variability of demand on conventional units. Through a dynamic game-theoretic formulation, we show that (under suitable convexity assumptions) the proposed pricing mechanism achieves social optimality asymptotically, as the number of consumers increases to infinity. Numerical results demonstrate that compared with marginal cost pricing, the proposed mechanism creates a stronger incentive for consumers to shift their peak load, and therefore has the potential to reduce the need for long-term investment in peaking plants.
\end{abstract}

Key words: Electricity market, dynamic pricing, social welfare

Date: November 7, 2018

\section{Introduction}

This paper is motivated by the fact that fluctuations in the demand for electricity to be met by conventional thermal generating units typically result in significantly increased, and nontrivial, ancillary costs. Today, such demand fluctuations are mainly due to time-dependent consumer preferences. In addition, in the future, a certain percentage of electricity production is required by law in many states in the U.S. to come from renewable resources (Barbose et al. 2008). The dramatic volatility of renewable energy resources may aggravate the variability of the demand for conventional thermal generators and result in significant ancillary cost. More concretely, either a demand surge or a decrease in renewable generation may result in (i) higher energy costs due 
to the deployment of peaking plants with higher ramping rates but higher marginal cost, such as oil/gas combustion turbines, and (ii) the redispatch cost $^{1}$ that the system will incur to meet reserve constraints if the increase of demand (or decrease of renewable generation) causes a reserve shortage.

There is general agreement that charging real-time prices (that reflect current operating conditions) to electricity consumers has the potential of reducing supplier ancillary cost, improving system efficiency, and lowering volatility in wholesale prices (US Department of Energy 2006, Spees and Lave 2008, Chao 2010). Therefore, dynamic pricing, especially real-time marginal cost pricing, is often identified as a priority for the implementation of wholesale electricity markets with responsive demand (Hogan 2010), which in turn raises many new questions. For example, should prices for a given time interval be calculated ex ante or ex post? Does real-time pricing introduce the potential for new types of market instabilities? How is supplier competition affected? In this paper, we abstract away from almost all of these questions and focus on the specific issue of whether prices should also explicitly encourage consumers to adapt their demands so as to reduce supplier ancillary cost.

To illustrate the issue that we focus on, we note that a basic model of electricity markets assumes that the cost of satisfying a given level $A_{t}$ of aggregate demand during period $t$ is of the form $C\left(A_{t}\right)$. It then follows that in a well-functioning wholesale market, the observed price should more or less reflect the marginal cost $C^{\prime}\left(A_{t}\right)$. In particular, prices should be more or less determined by the aggregate demand level. Empirical data do not quite support this view. Fig. 1 plots the real-time system load and the hourly prices on February 11, 2011 and on February 16, 2011, as reported by the New England ISO (ISO New England Inc. 2011). We observe that prices do not seem to be determined solely by $A_{t}$ but that the changes in demand, $A_{t}-A_{t-1}$, also play a major role. In particular, the largest prices seem to occur after a demand surge, and not necessarily at

\footnotetext{
${ }^{1}$ A certain level of reserve must always be maintained in an electric power system. Local reserve shortages are usually due to the quick increase of system load rather than a capacity deficiency. If the increase of system load makes the system short in reserves, the system will redispatch resources to increase the amount of reserves available. Redispatch generally increases the generation cost and results in higher prices. The redispatch cost can be very high (cf. Section 2.3.2 of ISO New England Inc. (2010)).
} 

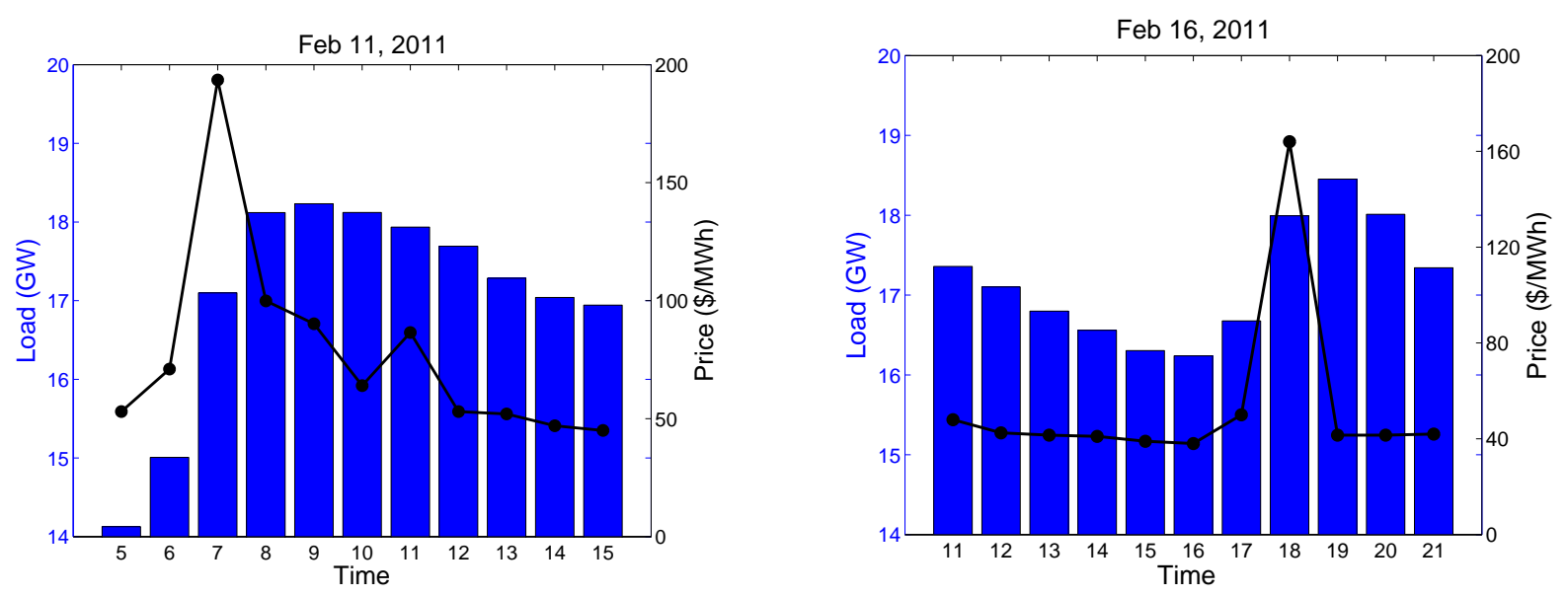

Figure 1 Real-time prices and actual system load, ISO New England Inc. Blue bars represent the real-time system loads and the dots connected by a black line represent the hourly prices.

the hour when the load is highest. We take this as evidence that the total cost over $T+1$ periods is not of the form

$$
\sum_{t=0}^{T} C\left(A_{t}\right)
$$

but rather of the form

$$
\sum_{t=0}^{T}\left(C\left(A_{t}\right)+H\left(A_{t-1}, A_{t}\right)\right)
$$

for a suitable function $H$.

We take the form of Eq. (1) as our starting point and raise the question of the appropriate prices. A naive view would argue that at time $t, A_{t-1}$ has already been realized, and taking its value for granted, a consumer should be charged a unit price equal to

$$
C^{\prime}\left(A_{t}\right)+\frac{\partial}{\partial A_{t}} H\left(A_{t-1}, A_{t}\right)
$$

which is the supplier's marginal cost at stage $t$. We refer to this naive approach as "marginal cost pricing" (MCP). However, a simple argument based on standard mathematical programming optimality conditions shows that for system optimality to obtain, the demand $A_{t-1}$ should also incur (after $A_{t}$ is realized) a unit price of

$$
\frac{\partial}{\partial A_{t-1}} H\left(A_{t-1}, A_{t}\right) .
$$


In day-ahead markets, suppliers typically carry out an intertemporal optimization, and it is reasonable to expect that the two types of marginal costs, captured by Eqs. (2) and (3), are both properly accounted for. However, in current real-time balancing markets, once $A_{t-1}$ is realized, a supplier will aim at charging the marginal cost in Eq. (2), but will be unable to charge the additional marginal cost in Eq. (3) to the past demand $A_{t-1}$. In contrast, the pricing mechanism that we propose and analyze in this paper is designed to include the additional marginal cost in Eq. $(3) .^{2}$

The actual model that we consider will be richer from the one discussed above in a number of respects. It includes an exogenous source of uncertainty (e.g., representing weather conditions) that has an impact on consumer utility and supplier cost, and therefore the model can incorporate the effects of volatile renewable electricity production. It allows for consumers with internal state variables (e.g., a consumer's demand may be affected by how much electricity she has already used). It also allows for multiple consumer types (i.e., with different utility functions and different internal state dynamics). Consumers are generally modeled as price-takers, as would be the case in a model involving an infinity (a continuum) of consumers. However, we also consider the case of finite consumer populations and explore certain equilibrium concepts that are well-suited to the case of finite but large consumer populations. On the other hand, we ignore most of the distinctions between ex post and ex ante prices. Instead, we assume that at each time step, the electricity market clears. The details of how this could happen are important, but are generic to electricity markets, hence not specific to our models, and somewhat orthogonal to the subject of this paper. (See however Appendix B for some discussion of implementation issues.)

The ancillary cost function $H\left(A_{t-1}, A_{t}\right)$ is of course a central element of our model. How can we be sure that this is the right form? In general, redispatch and reserve dynamics are complicated and one should not expect such a function to capture all of the complexity of the true system costs;

\footnotetext{
${ }^{2}$ In current two-settlement systems, the real-time prices are charged only on the difference of the actual demand and the estimated demand at the day-ahead market. However, the two-settlement system provides the same real-time incentives to price-taking consumers, as if they were purchasing all of their electricity at the real-time prices (cf. Chapter 3-2 of Stoft (2002)).
} 
perhaps, a more complex functional form such as $H\left(A_{t-2}, A_{t-1}, A_{t}\right)$ would be more appropriate. We believe that the form we have chosen is a good enough approximation, at least under certain conditions. To argue this point, we present in Appendix A an example that involves a more detailed system model (in which the true cost is a complicated function of the entire history of demands) and show that a function of the form $H\left(A_{t-1}, A_{t}\right)$ can capture most of the cost of ancillary services.

\subsection{Summary of contributions}

Before continuing, we provide here a roadmap of the paper together with a summary of our main contributions.

(a) We provide a stylized (yet quite rich) model of an electricity market, which incorporates the cost of ancillary services (cf. Section 2).

(b) We provide some justification of the form of the cost function in our model, as a reasonable approximation of more detailed physical models (cf. Appendix A).

(c) We propose a pricing mechanism that properly charges for the effects of consumer actions on ancillary services (cf. Section 3).

(d) For a continuum model involving nonatomic price-taking consumers, we consider Dynamic Oblivious Equilibria (DOE), in which every consumer maximizes her expected payoff under the sequence of prices induced by a DOE strategy profile (Section 4). We show that (under standard convexity assumptions), our mechanism maximizes social welfare (cf. Theorem 2 in Section 6).

(e) We carry out a game-theoretic analysis of the case of a large but finite number of consumers. We show that a large population of consumers who act according to a DOE (derived from an associated continuum game) results in asymptotically optimal (as the number of consumers goes to infinity) social welfare (cf. Theorem 2 in Section 6), and asymptotically maximizes every consumer's expected payoff (this is an "asymptotic Markov equilibrium" property; cf. Theorem 1 in Section $5)$.

(f) We illustrate the potential benefits of our mechanism through a simple numerical example. In particular, we show that compared with marginal cost pricing, the proposed mechanism reduces 
the peak load, and therefore has the potential to reduce the need for long-term investments in peaking plants (cf. Appendix E).

\subsection{Related literature}

There are two streams of literature, on electricity pricing and on game theory, that are relevant to our work, and which we now proceed to discuss, while also highlighting the differences from the present work.

Regarding electricity markets, the impact of supply friction on economic efficiency and price volatility has received some recent attention. Mansur (2008) shows that under ramping constraints, the prices faced by consumers may not necessarily equal the true supplier marginal cost. In a continuous-time competitive market model, Cho and Meyn (2010a) show that the limited capability of generating units to meet real-time demand, due to relatively low ramping rates, does not harm social welfare, but may result in extreme price fluctuations. In a similar spirit, Kizilkale and Mannor (2010) construct a dynamic game-theoretic model to study the tradeoff between economic

efficiency and price volatility. Closer to the present paper, Cho and Meyn (2010b) construct a dynamic newsboy model to study the reserve management problem in electricity markets, where the demand is assumed to be exogenous. The supplier cost in their model depends not only on the overall demand, but also on the generation resources used to satisfy the demand. For example, a quickly increasing demand may require more responsive and more expensive resources (e.g., peaking generation plants).

To study the impact of pricing mechanisms on consumer behavior and load fluctuations, we construct a dynamic game-theoretic model that differs from existing dynamic models for electricity markets and incorporates both the consumers' responses to real-time price fluctuations and the suppliers' ancillary cost incurred by load swings. Some major differences between our model and existing ones are discussed at the end of Section 2.

On the game-theoretic side, the standard solution concept for stochastic dynamic games is the Markov perfect equilibrium (MPE) (Fudenberg and Tirole 1991, Maskin and Tirole 1988), 
involving strategies where an agent's action depends on the current state of all agents. As the number of agents grows large, the computation of an MPE is often intractable (Doraszelski and Pakes 2007). For this reason, alternative equilibrium concepts, for related games featuring a nonatomic continuum of agents (e.g., "oblivious equilibrium" or "stationary equilibrium" for dynamic games without aggregate shocks), have received much recent attention (Weintraub et al. 2009, Adlakha et al. 2011).

There is a large literature on a variety of approximation properties of nonatomic equilibria (MasColell and Vives 1993, Al-Najjar 2004, 2008). Recently, Adlakha et al. (2011) derive sufficient conditions for a stationary equilibrium strategy to have the Asymptotic Markov Equilibrium (AME) property, i.e., a stationary equilibrium strategy asymptotically maximizes every agent's expected payoff (given that all the other agents use the same stationary equilibrium strategy), as the number of agents grows large. Their model includes random shocks that are assumed to be idiosyncratic across agents. However, in the problem that we are interested in, it is important to incorporate aggregate shocks (such as weather conditions) that have a global impact on all agents. In this spirit, Weintraub et al. (2010) consider a market model with aggregate profit shocks, and study an equilibrium concept at which every firm's strategy depends on the firm's current state and on the recent history of the aggregate shock. For a general dynamic game model with aggregate shocks, Bodoh-Creed (2010) shows that a nonatomic counterpart of an MPE, which we refer to as a Dynamic Oblivious Equilibrium (DOE) in this paper, asymptotically approximates an MPE in the sense that as the number of agents increases to infinity, the actions taken in an MPE can be well approximated by those taken by a DOE strategy of the nonatomic limit game. However, without further restrictive assumptions on the agents' state transition kernel, the approximation property of the actions taken by a DOE strategy does not imply the AME property of the DOE, and we are not aware of any AME results for models that include aggregate shocks. Our work is different in this respect: for a dynamic nonatomic model with aggregate shocks, which is a simplified variation of the general model considered in Bodoh-Creed (2010), we prove the AME property of a DOE. 
The efficiency of nonatomic equilibria for static games has been addressed in recent research (Roughgarden and Tardos 2004, Milchtaich 2004, Bodoh-Creed 2011). For a dynamic industry model with a continuum of identical producers and exogenous aggregate shocks, Lucas and Prescott (1971) show (under convexity assumptions) that the expected social welfare is maximized at a unique competitive equilibrium. In a similar spirit, in this paper we show (under convexity assumptions) that the proposed pricing mechanism maximizes the expected social welfare in a model involving a continuum of (possibly heterogeneous) consumers. We also consider the case of a large but finite number of consumers, and show that the expected social welfare is approximately maximized if all consumers act according to a nonatomic equilibrium (DOE). For large dynamic games, the asymptotic social optimality of nonatomic equilibria (DOEs) established in this paper seems to be new.

\section{Model}

We consider a $(T+1)$-stage dynamic game with the following elements:

1. The game is played in discrete time. We index the time periods with $t=0,1, \ldots, T$. Each stage may represent a five minute interval in real-time balancing markets where prices and dispatch solutions are typically provided at five minute intervals.

2. There are $n$ consumers, indexed by $1, \ldots, n$.

3. At each stage $t$, let $s_{t} \in \mathcal{S}$ be an exogenous state, which evolves as a Markov chain and whose transitions are not affected by consumer actions. The set $\mathcal{S}$ is assumed to be finite. In electricity markets, the exogenous state may represent time and/or weather conditions, which impact consumer utility and supplier cost. It may also represent the level of renewable generation.

4. For notational conciseness, for $t \geq 1$, let $\bar{s}_{t}=\left(s_{t-1}, s_{t}\right)$, and let $\bar{s}_{0}=s_{0}$. We use $\overline{\mathcal{S}}_{t}$ to denote the set of all possible $\bar{s}_{t}$. We refer to $\bar{s}_{t}$ as the global state at stage $t$.

5. Given an initial global state $s_{0}$, the initial states (types) of the consumers, $\left\{x_{i, 0}\right\}_{i=1}^{n}$, are independently drawn according to a probability measure $\eta_{s_{0}}$ over a finite set $\mathcal{X}_{0}$. We use $X$ to denote the cardinality of $\mathcal{X}_{0}$. 
6. The state of consumer $i$ at stage $t$ is denoted by $x_{i, t}$. At $t=0$, consumer $i$ 's initial state, $x_{i, 0}$, indicates her type. For $t=1, \ldots, T$, we have $x_{i, t}=\left(x_{i, 0}, z_{i, t}\right)$, where $z_{i, t} \in \mathcal{Z}$ and $\mathcal{Z}=[0, Z]$ is a compact subset of $\mathbb{R}$. The variables $\left\{z_{i, t}\right\}_{i=1}^{n}$ allow us to model intertemporal substitution effects in consumer $i$ 's demand.

7. We use $\mathcal{X}_{t}$ to denote a consumer's state space at stage $t$. In particular, at stage $t \geq 1$, $\mathcal{X}_{t}=\mathcal{X}_{0} \times \mathcal{Z}$

8. At stage $t$, consumer $i$ takes an action $a_{i, t}$ and receives a nonnegative utility $U_{t}\left(x_{i, t}, s_{t}, a_{i, t}\right)$.

9. Each consumer's action space is $\mathcal{A}=[0, B]$, where $B$ is a positive real number. (In the electric power context, $B$ could reflect a local transmission capacity constraint.)

10. We use $A_{t}=\sum_{i=1}^{n} a_{i, t}$ to denote the aggregate demand at stage $t$.

11. Given consumer $i$ 's current state, $x_{i, t}$, and the next exogenous state $s_{t+1}$, the next state of consumer $i$ is determined by her action taken at stage $t$, i.e., $x_{i, t+1}=\left(x_{i, 0}, z_{i, t+1}\right)$, where $z_{i, t+1}=$ $r\left(x_{i, t}, a_{i, t}, s_{t+1}\right)$, for a given function $r$.

12. Let $G_{t}=A_{t}+R_{t}$ be the capacity available at stage $t$, where $R_{t}$ is the system reserve at stage $t$. For simplicity, we assume that the system reserve at stage $t$ depends only on the current aggregate demand, $A_{t}$, and the current exogenous state $s_{t}$. That is, we have $R_{t}=g\left(A_{t}, s_{t}\right)$ for a given function of $g$ that reflects the reserve policy of the system operator.

13. At stage $t$, let $\bar{C}\left(A_{t}, R_{t}, s_{t}\right)$ be the total conventional generation cost, that is, the sum of the supplier's cost to meet the aggregate demand $A_{t}$ through its primary energy resources, e.g., base-load power plants, and the cost to maintain a system reserve $R_{t}$. Since $R_{t}$ depends only on $A_{t}$ and $s_{t}$, we can write $\bar{C}\left(A_{t}, R_{t}, s_{t}\right)$ as a function of $A_{t}$ and $s_{t}$, i.e., there exists a primary cost function $C: \mathbb{R} \times \mathcal{S} \rightarrow[0, \infty)$ such that $C\left(A_{t}, s_{t}\right)=\bar{C}\left(A_{t}, R_{t}, s_{t}\right)$. We assume that for any $s \in \mathcal{S}$, $C(\cdot, s)$ is nondecreasing.

14. At stage $t \geq 1$, let $\bar{H}\left(A_{t-1}, A_{t}, R_{t-1}, R_{t}, s_{t}\right)$ denote the ancillary cost incurred by load swings ${ }^{4}$.

${ }^{3}$ At $t=0, U_{0}$ is a mapping from $\mathcal{X}_{0} \times \mathcal{S} \times \mathcal{A}$ to $[0, \infty)$, while for $t \geq 1, U_{t}$ is a mapping from $\mathcal{X}_{0} \times \mathcal{Z} \times \mathcal{S} \times \mathcal{A}$ to $[0, \infty)$. ${ }^{4}$ In general, the supplier ancillary cost may depend on the entire history of system load and global states. However, ancillary cost functions with the simple form $\bar{H}\left(A_{t-1}, A_{t}, R_{t-1}, R_{t}, s_{t}\right)$ can serve as a good approximation of the supplier's true ancillary cost (cf. Appendix A). 
Since $R_{t}$ depends only on $A_{t}$ and $s_{t}$, we can write $\bar{H}\left(A_{t-1}, A_{t}, R_{t-1}, R_{t}, s_{t}\right)$ as a function of $A_{t-1}$, $A_{t}, s_{t-1}$, and $s_{t}$, i.e., there exists an ancillary cost function $H: \mathbb{R}^{2} \times \mathcal{S}^{2} \rightarrow[0, \infty)$ such that $\bar{H}=H\left(A_{t-1}, A_{t}, \bar{s}_{t}\right)$. The ancillary cost at stage 0 is assumed to be a function of $s_{0}$ and $A_{0}$.

15. At stage 0 , the total supplier cost is of the form

$$
C\left(A_{0}, s_{0}\right)+H_{0}\left(A_{0}, s_{0}\right)
$$

and for $t=1, \ldots, T$, the total supplier cost at stage $t$ is given by

$$
C\left(A_{t}, s_{t}\right)+H\left(A_{t-1}, A_{t}, \bar{s}_{t}\right) .
$$

In contrast to existing dynamic models for electricity markets with an exogenous demand process (Cho and Meyn 2010a,b), our dynamic game-theoretic model incorporates the consumer reactions to price fluctuations, and allows us to study the impact of pricing mechanisms on consumer behavior and economic efficiency. Through a dynamic game-theoretic formulation, Kizilkale and Mannor (2010) study the tradeoff between economic efficiency and price volatility. Our model is different from the one studied in Kizilkale and Mannor (2010) in the following respects:

1. Our model allows the generation cost to depend on an exogenous state, and therefore can incorporate supply-side volatility due to uncertainty in renewable electricity generation. As an example, consider a case where the exogenous state, $s_{t}$, represents the electricity generation from renewable resources at stage $t$. Then the demand for conventional generation is $A_{t}-s_{t}$. Suppose that the system reserve is proportional to the system load, say, $\delta A_{t}$ for some constant $\delta>0$. The cost function, $\bar{C}\left(A_{t}, R_{t}, s_{t}\right)$, then depends only on the output of conventional generating units, $A_{t}-s_{t}$, and the system reserve, $\delta A_{t}$. The ancillary cost occurred at stage $t$ depends on the system reserve and the outputs of conventional generating units at stages $t-1$ and $t$, and is therefore a function of $A_{t-1}, A_{t}, s_{t-1}$, and $s_{t}$.

2. More important, instead of penalizing each consumer's attempt to change her own action across time, the ancillary cost function in our model penalizes the change in the aggregate demand by all consumers. The change in a single consumer's action may harm or benefit the social welfare, while the volatility of the aggregate demand is usually undesirable. 
The main feature of our model is the ancillary cost function $H$, which makes the supplier cost nonseparable over time. In an electric power system, the ancillary cost function models the costs associated with the variability of conventional thermal generator output, such as the energy cost of peaking plants and the redispatch cost. Note that the ancillary cost is not necessarily zero when $A_{t} \leq A_{t-1}$, because thermal generating units have ramping-down constraints, and because a decrease in renewable electricity production may lead to an increase of the system reserve, even if $A_{t} \leq A_{t-1}$. The presence of the ancillary cost function makes conventional marginal cost pricing inefficient (cf. Example 1 in Section 3).

To keep the model simple, we do not incorporate any idiosyncratic randomness in consumer state evolution. Thus, besides the randomness of consumer types (initial states), the only source of stochasticity in the model is the exogenous state $s_{t}$.

To effectively highlight the impact of pricing mechanisms on consumer behavior, as well as on economic efficiency and demand volatility, we have made the following simplifications and assumptions for the power grid:

(a) As in Cho and Meyn (2010a), we assume that the physical production capacity is large enough so that the possible changes of the generation capacity are not constrained.

(b) Transmission capacity is large enough to avoid any congestion. We also assume that the cost of supplying electricity to consumers at different locations is the same. Therefore, a common price for all consumers is appropriate.

(c) We use a simplified form of ancillary cost functions, $\bar{H}\left(A_{t-1}, A_{t}, R_{t-1}, R_{t}, s_{t}\right)$, to approximate the supplier ancillary cost. In Appendix A, we present a numerical example to justify this approximation.

\section{The Pricing Mechanism}

The marginal cost pricing mechanism discussed in Section 1 charges a time-varying unit price on each consumer's demand. As demonstrated in the following example, a time-varying price that equals the supplier's instantaneous marginal cost may not achieve social optimality in a setting 
that includes ancillary costs. For this reason, we propose a new pricing mechanism that takes into account the ancillary cost associated with a consumer's demand at the previous stage.

ExAmple 1. Consider a two-stage deterministic model with one consumer and one supplier. At stage $t$, the consumer's utility function is $U_{t}:[0, \infty) \rightarrow[0, \infty)$. Let $a_{t}$ denote the demand at stage $t$, and let $\mathbf{a}=\left(a_{0}, a_{1}\right)$. Let $g_{t}$ denote the actual generation at stage $t$, and let $\mathbf{g}=\left(g_{0}, g_{1}\right)$. Two unit prices, $p_{0}$ and $p_{1}$, are charged on the consumption at stage 0 and 1 , respectively. Let $\mathbf{p}=\left(p_{0}, p_{1}\right)$. The consumer's payoff-maximization problem is

$$
\underset{\mathbf{a}}{\operatorname{Maximize}} U_{0}\left(a_{0}\right)-p_{0} a_{0}+U_{1}\left(a_{1}\right)-p_{1} a_{1}
$$

Let $H_{0}$ be identically zero, and let the ancillary cost function at stage 1 depend only on the difference between the supply at the two stages. That is, the ancillary cost at stage 1 is of the form $H\left(g_{1}-g_{0}\right)$. The supplier's profit-maximization problem is

$$
\underset{\mathbf{g}}{\operatorname{Maximize}} p_{0} g_{0}+p_{1} g_{1}-C\left(g_{0}\right)-C\left(g_{1}\right)-H\left(g_{1}-g_{0}\right)
$$

The social planner's problem is

$$
\begin{array}{ll}
\underset{(\mathbf{a}, \mathbf{g})}{\operatorname{Maximize}} & U_{0}\left(a_{0}\right)+U_{1}\left(a_{1}\right)-C\left(g_{0}\right)-C\left(g_{1}\right)-H\left(g_{1}-g_{0}\right) \\
\text { subject to } & \mathbf{a}=\mathbf{g} .
\end{array}
$$

Now consider a competitive equilibrium, $(\mathbf{a}, \mathbf{g}, \mathbf{p})$, at which the vector a solves the consumer's optimization problem (6), the vector $\mathbf{g}$ solves the supplier's optimization problem (7), and the market clears, i.e., $\mathbf{a}=\mathbf{g}$. Suppose that the utility functions are concave and continuously differentiable, and that the cost functions $C$ and $H$ are convex and continuously differentiable. We further assume that $H^{\prime}(0)=0$, and that for $t=0,1, U_{t}^{\prime}(0)>C^{\prime}(0), U_{t}^{\prime}(B)<C^{\prime}(B)$. Then, there exists a competitive equilibrium, $(\mathbf{a}, \mathbf{g}, \mathbf{p})$, which satisfies the following conditions:

$$
\left\{\begin{array} { l } 
{ U _ { 0 } ^ { \prime } ( a _ { 0 } ) = p _ { 0 } , } \\
{ U _ { 1 } ^ { \prime } ( a _ { 1 } ) = p _ { 1 } , }
\end{array} \quad \left\{\begin{array}{l}
C^{\prime}\left(a_{0}\right)-H^{\prime}\left(a_{1}-a_{0}\right)=p_{0}, \\
C^{\prime}\left(a_{1}\right)+H^{\prime}\left(a_{1}-a_{0}\right)=p_{1} .
\end{array}\right.\right.
$$

We conclude that the competitive equilibrium solves the social welfare maximization problem in (8), because it satisfies the following (sufficient) optimality conditions:

$$
\begin{aligned}
& U_{0}^{\prime}\left(a_{0}\right)=C^{\prime}\left(a_{0}\right)-H^{\prime}\left(a_{1}-a_{0}\right), \quad U_{1}^{\prime}\left(a_{1}\right)=C^{\prime}\left(a_{1}\right)+H^{\prime}\left(a_{1}-a_{0}\right), \\
& a_{0}=g_{0}, \quad a_{1}=g_{1} .
\end{aligned}
$$


However, we observe that the socially optimal price $p_{0}$ does not equal the supplier's instantaneous marginal cost at stage $0, C^{\prime}\left(a_{0}\right)$. Hence, by setting the price equal to $C^{\prime}\left(a_{0}\right)$, as would be done in a real-time balancing market, we may not achieve social optimality. More generally, marginal cost pricing need not be socially optimal because it does not take into account the externality conferred by the action $a_{0}$ on the ancillary cost at stage $1, H\left(a_{1}-a_{0}\right)$. At a socially optimal competitive equilibrium, the consumer should pay

$$
\left(C^{\prime}\left(a_{0}\right)-H^{\prime}\left(a_{1}-a_{0}\right)\right) a_{0}+\left(C^{\prime}\left(a_{1}\right)+H^{\prime}\left(a_{1}-a_{0}\right)\right) a_{1},
$$

i.e., the price on $a_{0}$ should be the sum of the supplier marginal cost at stage $0, C^{\prime}\left(a_{0}\right)$, and the marginal ancillary cost associated with $a_{0},-H^{\prime}\left(a_{1}-a_{0}\right)$, which is determined at the next stage, after $a_{1}$ is realized.

Before describing the precise pricing mechanism we propose, we introduce a differentiability assumption on the cost functions.

Assumption 1. For any $s \in \mathcal{S}, C(\cdot, s)$ and $H_{0}(\cdot, s)$ are continuously differentiable on $[0, \infty)$. For any $\left(A^{\prime}, \bar{s}\right) \in \mathcal{A} \times \mathcal{S}^{2}, H\left(A, A^{\prime}, \bar{s}\right)$ and $H\left(A^{\prime}, A, \bar{s}\right)$ are continuously differentiable in $A$ on $[0, \infty) .{ }^{5}$

Inspired by Example 1, we introduce prices

$$
p_{t}=C^{\prime}\left(A_{t}, s_{t}\right), \quad t=0, \ldots, T,
$$

and

$$
q_{t}=\frac{\partial H\left(A_{t-1}, A_{t}, \bar{s}_{t}\right)}{\partial A_{t-1}}, \quad w_{t}=\frac{\partial H\left(A_{t-1}, A_{t}, \bar{s}_{t}\right)}{\partial A_{t}}, \quad t=1, \ldots, T
$$

At stage 0 , we let $q_{0}=0$ and $w_{0}=H_{0}^{\prime}\left(A_{0}, s_{0}\right)$. Under the proposed pricing mechanism, consumer $i$ 's payoff at stage $t$ is given by

$$
U_{t}\left(x_{i, t}, s_{t}, a_{i, t}\right)-\left(p_{t}+w_{t}\right) a_{i, t}-q_{t} a_{i, t-1}
$$

\footnotetext{
${ }^{5}$ At the boundary of the domain, 0, we require continuity of the right-derivatives of $C, H_{0}$, and $H$.
} 
Note that $p_{t}+w_{t}$ is the supplier marginal cost at stage $t$ (including the marginal ancillary cost). The proposed pricing mechanism charges consumer $i$ an additional price $q_{t}$ on her previous demand, equal to the marginal ancillary cost with respect to $a_{i, t-1}$.

We now define some of the notation that we will be using. For $t=1, \ldots, T$, let $y_{i, t}=\left(a_{i, t-1}, x_{i, t}\right)$ be the augmented state of consumer $i$ at stage $t$. At $t=0$, let $y_{i, 0}=x_{i, 0}$. For stage $t$, let $\mathcal{Y}_{t}$ be the set of all possible augmented states. In particular, we have $\mathcal{Y}_{0}=\mathcal{X}_{0}$, and $\mathcal{Y}_{t}=\mathcal{A} \times \mathcal{X}_{t}$, for $t=1, \ldots, T$.

Let $\Delta_{n}(D)$ be the set of empirical probability distributions over a given set $D$ that can be generated by $n$ samples from $D$. (Note that empirical distributions are always discrete, even if $D$ is a continuous set.) Let $f_{t} \in \Delta_{n}\left(\mathcal{Y}_{t}\right)$ be the empirical distribution of the augmented state of all consumers at stage $t$, and let $f_{-i, t} \in \Delta_{n-1}\left(\mathcal{Y}_{t}\right)$ be the empirical distribution of the augmented state of all consumers (excluding consumer $i$ ) at stage $t$. We refer to $f_{t}$ as the population state at stage $t$. Let $u_{t} \in \Delta_{n}(\mathcal{A})$ denote the empirical distribution of all consumers' actions at stage $t$, and let $u_{-i, t} \in \Delta_{n-1}(\mathcal{A})$ be the empirical distribution of all consumers' (excluding consumer $i$ ) actions at stage $t$.

For a given $n$, it can be seen from (11) and (12) that the prices, and thus the stage payoff in (13), are determined by the current global state, $\bar{s}_{t}$, consumer $i$ 's current augmented state, $y_{i, t}$, and current action, $a_{i, t}$, as well as the empirical distributions, $f_{-i, t}$ and $u_{-i, t}$ of other consumers' current augmented state and action. Hence, for a certain function $\pi(\cdot)$, we can write the stage payoff in (13) as

$$
\pi\left(y_{i, t}, \bar{s}_{t}, a_{i, t}, f_{-i, t}, u_{-i, t}\right)=U_{t}\left(x_{i, t}, s_{t}, a_{i, t}\right)-\left(p_{t}+w_{t}\right) a_{i, t}-q_{t} a_{i, t-1} .
$$

\section{A Continuum Model and Dynamic Oblivious Strategies}

To study the aggregate behavior of a large number of consumers, we consider a nonatomic game involving a continuum of infinitesimally small consumers, indexed by $i \in[0,1]$. We assume that (under state $s_{0}$ ) a fraction $\eta_{s_{0}}$ of the consumers has initial state $x$. In a nonatomic model, any single consumer's action has no influence on the aggregate demand and the prices. We consider a 
class of strategies (dynamic oblivious strategies) in which a consumer's action depends only on the history of past exogenous states, $h_{t}=\left(s_{0}, \ldots, s_{t}\right)$, and her own current state ${ }^{6}$, i.e., of the form

$$
a_{i, t}=\bar{\nu}_{t}\left(x_{i, t}, h_{t}\right)
$$

Suppose that consumer $i$ uses a dynamic oblivious strategy $\bar{\nu}=\left(\bar{\nu}_{0}, \ldots, \bar{\nu}_{T}\right)$. Since there is no idiosyncratic randomness, given a history $h_{t}$, the state $x_{i, t}$ of consumer $i$ at stage $t$ depends only on her initial state $x_{i, 0}$. That is, there is a mapping $l_{\bar{\nu}, h_{t}}: \mathcal{X}_{0} \rightarrow \mathcal{X}_{t}$, such that $x_{i, t}=l_{\bar{\nu}, h_{t}}\left(x_{i, 0}\right)$.

Therefore, we can specify the action taken by a dynamic oblivious strategy in the alternative form

$$
a_{i, t}=\nu_{t}\left(x_{i, 0}, h_{t}\right) \triangleq \bar{\nu}_{t}\left(l_{\bar{\nu}, h_{t}}\left(x_{i, 0}\right), h_{t}\right) .
$$

We refer to $\nu=\left(\nu_{0}, \ldots, \nu_{T}\right)$ as a dynamic oblivious strategy, and let $\mathfrak{V}$ be the set of all such strategies.

An alternative formulation involving strategies that depend on consumer expectations on future prices would lead to a Rational Expectations Equilibrium (REE), an equilibrium concept based on the rational expectations approach pioneered by Muth (1961). In our continuum model, since the only source of stochasticity is from the exogenous state $s_{t}$, future prices under any given strategy profile, are completely determined by the history $h_{t}$. Therefore, it is reasonable to expect that strategies of the form (15) will lead to an equilibrium concept that is identical in outcomes with a REE (cf. the discussion in Section 4.2).

Before formally defining a Dynamic Oblivious Equilibrium (DOE), we first provide some of the intuition behind the definition. In a continuum model, if all consumers use a common dynamic oblivious strategy $\nu$, the aggregate demand and the prices at stage $t$ depend only on the history of exogenous states, $h_{t}=\left(s_{0}, \ldots, s_{t}\right)$. A dynamic oblivious strategy $\nu$ is a DOE (cf. the formal definition in Section 4.2) if it maximizes every consumer's expected total payoff, under the sequence

\footnotetext{
${ }^{6}$ Note that a dynamic oblivious strategy depends only on the consumer's current state, instead of her augmented state. As we will see in Section 4.2, in a continuum model, since any single consumer has no influence on the prices, a best response or equilibrium strategy need not take into account the action taken at the previous stage.
} 
of prices that $\nu$ induces. In Section 4.3, we associate a continuum model with a sequence of $n$ consumer models $(n=1,2, \ldots)$, and specify the relation between the continuum model and the corresponding $n$-consumer model.

\subsection{The sequence of prices induced by a dynamic oblivious strategy}

Let $h_{t}=\left(s_{0}, \ldots, s_{t}\right)$ denote a history up to stage $t$, and let $\mathcal{H}_{t}=\mathcal{S}^{t+1}$ denote the set of all possible such histories. Recall that in a continuum model, given an initial global state $s_{0}$, the distribution of consumers' initial states is $\eta_{s_{0}}$. Therefore, under a history $h_{t}$, if all consumers use the same dynamic oblivious strategy $\nu$, then the average demand is

$$
\widetilde{A}_{t \mid \nu, h_{t}}=\sum_{x \in \mathcal{X}_{0}} \eta_{s_{0}}(x) \cdot \nu_{t}\left(x, h_{t}\right)
$$

We now introduce the cost functions in a continuum model. Let $\widetilde{C}: \mathbb{R} \times \mathcal{S} \rightarrow[0, \infty)$ be a primary cost function. Let $\widetilde{H}: \mathbb{R}^{2} \times \mathcal{S}^{2} \rightarrow[0, \infty)$ be an ancillary cost function at stage $t \geq 1$, and let $\widetilde{H}_{0}$ : $\mathbb{R} \times \mathcal{S} \rightarrow[0, \infty)$ be an ancillary cost function at the initial stage 0.

Given the cost functions in a continuum model, we define the sequence of prices induced by a dynamic oblivious strategy as follows:

$$
\widetilde{p}_{t \mid \nu, h_{t}}=\widetilde{C}^{\prime}\left(\widetilde{A}_{t \mid \nu, h_{t}}, s_{t}\right), \quad \widetilde{q}_{0 \mid \nu, h_{0}}=0, \quad \widetilde{w}_{0 \mid \nu, h_{0}}=\widetilde{H}_{0}^{\prime}\left(\widetilde{A}_{0 \mid \nu, h_{0}}, s_{0}\right),
$$

and for $t \geq 1$,

$$
\widetilde{q}_{t \mid \nu, h_{t}}=\frac{\partial \widetilde{H}\left(\widetilde{A}_{t-1 \mid \nu, h_{t-1}}, \widetilde{A}_{t \mid \nu, h_{t}}, \bar{s}_{t}\right)}{\partial \widetilde{A}_{t-1 \mid \nu, h_{t-1}}}, \quad \widetilde{w}_{t \mid \nu, h_{t}}=\frac{\partial \widetilde{H}\left(\widetilde{A}_{t-1 \mid \nu, h_{t-1}}, \widetilde{A}_{t \mid \nu, h_{t}}, \bar{s}_{t}\right)}{\partial \widetilde{A}_{t \mid \nu, h_{t}}} .
$$

\subsection{Equilibrium strategies}

In this subsection we define the concept of a DOE. Suppose that all consumers other than $i$ use a dynamic oblivious strategy $\nu$. In a continuum model, consumer $i$ 's action does not affect the prices. If all consumers except $i$ use a dynamic oblivious strategy $\nu$, consumer $i$ 's oblivious stage value (the stage payoff in a continuum model) under a history $h_{t}$ and an action $a_{i, t}$, is

$$
\widetilde{\pi}_{i, t}\left(y_{i, t}, h_{t}, a_{i, t} \mid \nu\right)=U_{t}\left(x_{i, t}, s_{t}, a_{i, t}\right)-\left(\widetilde{p}_{t \mid \nu, h_{t}}+\widetilde{w}_{t \mid \nu, h_{t}}\right) a_{i, t}-\widetilde{q}_{t \mid \nu, h_{t}} a_{i, t-1}
$$


where the prices, $\widetilde{p}_{t \mid \nu, h_{t}}, \widetilde{w}_{t \mid \nu, h_{t}}$, and $\widetilde{q}_{t \mid \nu, h_{t}}$, are defined in (17) and (18). Since a single consumer's action cannot influence $\widetilde{q}_{t}$, the last term in (19) is not affected by the action $a_{i, t}$, and the decision $a_{i, t}$ at stage $t$ need not take $a_{i, t-1}$ into account, but should take $\widetilde{q}_{t+1}$ into account.

Consumer $i$ 's oblivious stage value under a dynamic oblivious strategy $\hat{\nu}$, is ${ }^{7}$

$$
\widetilde{\pi}_{i, t}\left(y_{i, t}, h_{t} \mid \hat{\nu}, \nu\right) \triangleq \widetilde{\pi}_{i, t}\left(y_{i, t}, h_{t}, \hat{\nu}_{t}\left(x_{i, 0}, h_{t}\right) \mid \nu\right)
$$

In particular, we use $\widetilde{\pi}_{i, t}\left(y_{i, t}, h_{t} \mid \nu, \nu\right)$ to denote the oblivious stage value of consumer $i$ at stage $t$, if all consumers use the strategy $\nu$. Given an initial global state $s_{0}$ and an initial state of consumer $i$, $x_{i, 0}$, her oblivious value function (total future expected payoff function in a continuum model) is

$$
\widetilde{V}_{i, 0}\left(x_{i, 0}, s_{0} \mid \hat{\nu}, \nu\right)=\mathbb{E}\left\{\sum_{\tau=0}^{T} \widetilde{\pi}_{i, \tau}\left(y_{i, \tau}, h_{\tau} \mid \hat{\nu}, \nu\right)\right\},
$$

where the expectation is over the future global states, $\left\{s_{\tau}\right\}_{\tau=1}^{T}$.

Definition 1. A strategy $\nu$ is a Dynamic Oblivious Equilibrium (DOE) if

$$
\sup _{\widehat{\nu} \in \mathfrak{V}} \widetilde{V}_{i, 0}\left(x_{i, 0}, s_{0} \mid \hat{\nu}, \nu\right)=\widetilde{V}_{i, 0}\left(x_{i, 0}, s_{0} \mid \nu, \nu\right), \quad \forall x_{i, 0} \in \mathcal{X}_{0}, \quad \forall s_{0} \in \mathcal{S}
$$

A DOE is guaranteed to exist, under suitable assumptions, and this is known to be the case for our model (under our assumptions), and even for a more general model that includes idiosyncratic randomness (Bergin and Bernhardt 1992). The DOE, as defined above, is essentially the same concept as the "dynamic competitive equilibrium" studied in Bodoh-Creed (2010), which is defined as the nonatomic equivalent of an MPE, in a continuum model. At a DOE, the beliefs of all consumers on future prices are consistent with the equilibrium outcomes. Therefore, a DOE is identical in outcomes with a Rational Expectations Equilibrium (REE).

In future electricity markets, consumers may form rational expectations of future prices through an adaptive learning process, or they may receive price estimates from utilities and/or the independent system operator through advanced metering infrastructures. (In Appendix B, we provide

\footnotetext{
${ }^{7}$ Recall that the initial state (the type) of consumer $i, x_{i, 0}$, is included in its state $x_{i, t}$, for any $t$.
} 
some discussion of a possible implementation of the proposed real-time pricing mechanism.) If so, a REE (equivalently, a DOE) will be a plausible outcome of such a market. Furthermore, we will show (Theorem 2) that under the proposed pricing mechanism, and under certain convexity assumptions, a DOE is socially optimal for the continuum model.

For a dynamic market model with aggregate profit shocks, Weintraub et al. (2010) introduce a concept of "extended oblivious equilibrium" at which every firm's strategy depends on its current state and on the recent history (as opposed to the full history) of the aggregate shock. The extended oblivious equilibrium is computationally tractable; however, an equilibrium strategy may not be an approximate best response for every firm, even if the number of firms is large (cf. the error bounds derived in Section 8.3 of Weintraub et al. (2010)).

Note that the definition of a DOE strategy requires optimality (attaining the supremum in Definition 2) only along the equilibrium path (Bodoh-Creed 2010). Thus, a DOE is similar in spirit to the "self-confirming equilibria" in Fudenberg and Levine (1993) and the "subjective equilibria" in Kalai and Lehrer (1995), in which each agent forms correct beliefs about her opponents only along the equilibrium path.

\subsection{The $n$-consumer model associated with a continuum model}

We want the cost functions in a continuum model to approximate the cost functions in an $n$ consumer model. Since the continuum of consumers is described by distributions over $[0,1]$, the demand given in (16) can be regarded as the average demand per consumer. To capture this correspondence, we assume the following relation between the cost functions in a continuum model and their counterparts in a corresponding $n$-consumer model.

Assumption 2. For any $n \in \mathbb{N}$, any $s \in \mathcal{S}$, and any $\bar{s}$ in $\mathcal{S}^{2}$, we have

$$
C^{n}(A, s)=n \widetilde{C}\left(\frac{A}{n}, s\right), \quad H_{0}^{n}(A, s)=n \widetilde{H}_{0}\left(\frac{A}{n}, s\right), H^{n}\left(A, A^{\prime}, \bar{s}\right)=n \widetilde{H}\left(\frac{A}{n}, \frac{A^{\prime}}{n}, \bar{s}\right)
$$

where the superscript $n$ is used to indicate that these are the cost functions associated with an n-consumer model. 
Assumption 2 implies that

$$
\left(C^{n}\right)^{\prime}(A, s)=\widetilde{C}^{\prime}(A / n, s), \quad\left(H_{0}^{n}\right)^{\prime}(A, s)=\widetilde{H}_{0}^{\prime}(A / n, s), \quad s \in \mathcal{S},
$$

and

$$
\frac{\partial H^{n}\left(A, A^{\prime}, \bar{s}\right)}{\partial A}=\frac{\partial \widetilde{H}\left(A / n, A^{\prime} / n, \bar{s}\right)}{\partial(A / n)}, \quad \frac{\partial H^{n}\left(A, A^{\prime}, \bar{s}\right)}{\partial A^{\prime}}=\frac{\partial \widetilde{H}\left(A / n, A^{\prime} / n, \bar{s}\right)}{\partial\left(A^{\prime} / n\right)}, \quad \forall \bar{s} \in \mathcal{S}^{2},
$$

i.e., the prices in the continuum model at the average demand equal the prices in the corresponding $n$-consumer model.

\section{Approximation in Large Games}

In this section, we consider a sequence of dynamic games, and show that as the number of consumers increases to infinity, a DOE strategy for the corresponding continuum game is asymptotically optimal for every consumer (i.e., an approximate best response), if the other consumers follow that same strategy. In the rest of the paper, we often use a superscript $n$ to indicate quantities associated with an $n$-consumer model.

Suppose that all consumers except $i$ use a dynamic oblivious strategy $\nu$. Given a history $h_{t}$ and an empirical distribution $f_{-i, t}^{n}$, we use $v\left(h_{t}, f_{-i, t}^{n}, \nu\right)$ to denote the empirical distribution, $u_{-i, t}^{n}$, of the actions taken by consumers excluding $i$. In an $n$-consumer model, suppose that consumer $i$ uses a history-dependent strategy $\kappa^{n}=\left\{\kappa_{t}^{n}\right\}_{t=0}^{T}$ of the form

$$
a_{i, t}=\kappa_{t}^{n}\left(y_{i, t}, h_{t}, f_{-i, t}^{n}\right),
$$

while the other consumers use a dynamic oblivious strategy $\nu$. Let $\mathfrak{K}_{n}$ denote the set of all possible history-dependent strategies $\kappa^{n}$ for the $n$-consumer model. Note that since all other consumers use an oblivious strategy $\nu, f_{-i, t}^{n}$ is completely determined by $\nu, f_{-i, 0}^{n}$, and $h_{t}$.

The stage payoff received by consumer $i$ at time $t$ is

$$
\pi_{i, t}^{n}\left(y_{i, t}, h_{t}, f_{-i, t}^{n} \mid \kappa^{n}, \nu\right)=\pi^{n}\left(y_{i, t}, \bar{s}_{t}, a_{i, t}, f_{-i, t}^{n}, v\left(h_{t}, f_{-i, t}^{n}, \nu\right)\right)
$$


where $a_{i, t}=\kappa_{t}^{n}\left(y_{i, t}, h_{t}, f_{-i, t}^{n}\right)$, and the stage payoff function on the right-hand side is given in (14). Given an initial global state, $s_{0}$, and consumer $i$ 's initial state, $x_{i, 0}$, consumer $i$ 's expected payoff under the strategy $\kappa^{n}$ is

$$
V_{i, 0}^{n}\left(x_{i, 0}, s_{0} \mid \kappa^{n}, \nu\right)=\mathbb{E}\left\{\sum_{t=0}^{T} \pi_{i, t}^{n}\left(y_{i, t}, h_{t}, f_{-i, t}^{n} \mid \kappa^{n}, \nu\right)\right\},
$$

where the expectation is over the initial distribution $f_{-i, 0}^{n}$ and over the future global states, $\left\{s_{t}\right\}_{t=1}^{T}$. In particular, we use $V_{i, 0}^{n}\left(x_{i, 0}, s_{0} \mid \nu, \nu\right)$ to denote the expected payoff obtained by consumer $i$ if all consumers use the strategy $\nu$.

Definition 2. A dynamic oblivious strategy $\nu$ has the asymptotic Markov equilibrium (AME) property (Adlakha et al. 2011), if for any initial global state $s_{0} \in \mathcal{S}$, any initial consumer state $x_{i, 0} \in \mathcal{X}_{0}$, and any sequence of history-dependent strategies $\left\{\kappa^{n}\right\}$, we have

$$
\limsup _{n \rightarrow \infty}\left(V_{i, 0}^{n}\left(x_{i, 0}, s_{0} \mid \kappa^{n}, \nu\right)-V_{i, 0}^{n}\left(x_{i, 0}, s_{0} \mid \nu, \nu\right)\right) \leq 0
$$

We will show that every DOE has the AME property, under the following assumption, which strengthens Assumption 1.

Assumption 3. We assume that:

3.1. The following four families of functions, of $A,\left\{\widetilde{C}^{\prime}(A, s): s \in \mathcal{S}\right\},\left\{\widetilde{H}_{0}^{\prime}(A, s): s \in \mathcal{S}\right\}$, $\left\{\partial \widetilde{H}\left(A, A^{\prime}, \bar{s}\right) / \partial A:\left(A^{\prime}, \bar{s}\right) \in \mathcal{A} \times \mathcal{S}^{2}\right\}$, and $\left\{\partial \widetilde{H}\left(A^{\prime}, A, \bar{s}\right) / \partial A:\left(A^{\prime}, \bar{s}\right) \in \mathcal{A} \times \mathcal{S}^{2}\right\}$, are uniformly equicontinuous on $[0, \infty) .^{8}$

3.2. The marginal costs are bounded from above, i.e.,

$$
\left|\widetilde{C}^{\prime}(A, s)\right| \leq P, \quad\left|\widetilde{H}_{0}^{\prime}(A, s)\right| \leq P, \quad \forall(A, s) \in \mathcal{A} \times \mathcal{S}
$$

and

$$
\left|\frac{\partial \widetilde{H}\left(A, A^{\prime}, \bar{s}\right)}{\partial A}\right| \leq P, \quad\left|\frac{\partial \widetilde{H}\left(A^{\prime}, A, \bar{s}\right)}{\partial A}\right| \leq P, \quad \forall\left(A^{\prime}, \bar{s}\right) \in \mathcal{A} \times \mathcal{S}^{2},
$$

where $P$ is a positive constant.

${ }^{8} \mathrm{~A}$ sufficient condition for this assumption to hold is to require a universal bound on the derivatives of the functions in each family. 
3.3. The utility functions, $\left\{U_{t}(x, s, a)\right\}_{t=0}^{T}$, are continuous in a and bounded above, i.e.,

$$
U_{t}(x, s, a) \leq Q, \quad t=0, \ldots, T, \quad \forall(x, s, a) \in \mathcal{X}_{t} \times \mathcal{S} \times \mathcal{A}
$$

where $Q$ is a positive constant.

Combining with Assumption 2, Assumption 3.1 implies that for any $\varepsilon>0$, there exists a $\delta>0$ such that for any positive integer $n$, if $|A-\bar{A}| \leq n \delta$, then

$$
\left|\left(C^{n}\right)^{\prime}(A, s)-\left(C^{n}\right)^{\prime}(\bar{A}, s)\right| \leq \varepsilon, \quad\left|\left(H_{0}^{n}\right)^{\prime}(A, s)-\left(H_{0}^{n}\right)^{\prime}(\bar{A}, s)\right| \leq \varepsilon, \quad \forall s \in \mathcal{S},
$$

and for any $\left(A^{\prime}, \bar{s}\right) \in \mathcal{A} \times \mathcal{S}^{2}$,

$$
\left|\frac{\partial H^{n}\left(A, A^{\prime}, \bar{s}\right)}{\partial A}-\frac{\partial H^{n}\left(\bar{A}, A^{\prime}, \bar{s}\right)}{\partial \bar{A}}\right| \leq \varepsilon, \quad\left|\frac{\partial H^{n}\left(A^{\prime}, A, \bar{s}\right)}{\partial A}-\frac{\partial H^{n}\left(A^{\prime}, \bar{A}, \bar{s}\right)}{\partial \bar{A}}\right| \leq \varepsilon
$$

Note that the boundness of the cost function derivatives implies the Lipschitz continuity of the cost functions. Combining with Assumption 2, for any pair of real numbers $(A, \bar{A})$, and any positive integer $n$, we have

$$
\left|C^{n}(A, s)-C^{n}(\bar{A}, s)\right| \leq P|A-\bar{A}|, \quad\left|H_{0}^{n}(A, s)-H_{0}^{n}(\bar{A}, s)\right| \leq P|A-\bar{A}|, \quad \forall s \in \mathcal{S},
$$

and for any $\left(A^{\prime}, \bar{s}\right) \in \mathcal{A} \times \mathcal{S}^{2}$,

$$
\left|H^{n}\left(A, A^{\prime}, \bar{s}\right)-H^{n}\left(\bar{A}, A^{\prime}, \bar{s}\right)\right| \leq P|A-\bar{A}|, \quad\left|H^{n}\left(A^{\prime}, A, \bar{s}\right)-H^{n}\left(A^{\prime}, \bar{A}, \bar{s}\right)\right| \leq P|A-\bar{A}| .
$$

We argue in the following theorem that a DOE strategy approximately maximizes a consumer's expected payoff (among all possible history-dependent strategies) in a dynamic game with a large but finite number of consumers, if the other consumers also use that strategy.

TheOrem 1. Suppose that Assumptions 2-3 hold. Every DOE has the AME property.

Theorem 1 is proved in Appendix C. Various approximation properties of nonatomic equilibrium concepts in a continuum game have been investigated in previous works. Sufficient conditions for a stationary equilibrium (an equilibrium concept for a continuum game without aggregate 
uncertainty) to have the AME property are derived in Adlakha et al. (2011). For a continuum game with both idiosyncratic and aggregate uncertainties, Bodoh-Creed (2010) shows that as the number agents increases to infinity, the actions taken in an MPE can be well approximated by some DOE strategy of the nonatomic limit game. Note, however, that in a general $n$-consumer game, even if all consumers take an action that is close to the action taken by a DOE strategy of the nonatomic limit game, the population states and the prices in the $n$-consumer game can still be very different from their counterparts in the nonatomic limit game. Therefore, without further assumptions on the consumers' state transition kernel (e.g., continuous dependence of consumer states on their previous actions), the approximation property of a DOE on the action space does not necessarily imply the AME property of the DOE.

\section{Asymptotic Social Optimality}

In Section 6.1, we define the social welfare associated with an $n$-consumer model and with a continuum model. In Section 6.2, we show that for a continuum model, the social welfare is maximized (over all symmetric dynamic oblivious strategy profiles) at a DOE, and that for a sequence of $n$-consumer models, if all consumers use the DOE strategy of the corresponding continuum model, then the social welfare is asymptotically maximized, as the number of consumers increases to infinity.

\subsection{Social welfare}

In an $n$-consumer model, let $\mathbf{x}_{t}=\left(x_{1, t}, \ldots, x_{n, t}\right)$ and $\mathbf{a}_{t}=\left(a_{1, t}, \ldots, a_{n, t}\right)$ be the vectors of consumer states and actions, respectively, at stage $t$. Under the current global state $\bar{s}_{t}$, the social welfare realized at stage $t$ is

$$
W_{t}^{n}\left(\mathbf{x}_{t}, \bar{s}_{t}, \mathbf{a}_{t}\right)=-C^{n}\left(A_{t}, s_{t}\right)-H^{n}\left(A_{t-1}, A_{t}, \bar{s}_{t}\right)+\sum_{i=1}^{n} U_{t}\left(x_{i, t}, s_{t}, a_{i, t}\right), \quad t=1, \ldots, T,
$$

and at stage 0 , the social welfare is

$$
W_{t}^{n}\left(\mathbf{x}_{0}, s_{0}, \mathbf{a}_{0}\right)=-C^{n}\left(A_{0}, s_{0}\right)-H_{0}^{n}\left(A_{0}, s_{0}\right)+\sum_{i=1}^{n} U_{0}\left(x_{i, 0}, s_{0}, a_{i, 0}\right) .
$$


Because of the symmetry of the problem, the social welfare at stage $t$ depends on $\mathbf{x}_{t}$ and $\mathbf{a}_{t}$ only through the empirical distribution of state-action pairs. In particular, under a symmetric historydependent strategy profile $\boldsymbol{\kappa}^{n}=\left(\kappa^{n}, \ldots, \kappa^{n}\right)$ (cf. the definition of a history-dependent strategy in Eq. (22)), we can write the social welfare at time $t$ (with a slight abuse of notation) as $W_{t}^{n}\left(f_{t}^{n}, h_{t} \mid\right.$ $\left.\boldsymbol{\kappa}^{n}\right)$. Given an initial global state $s_{0}$ and an initial population state $f_{0}^{n}$, the expected social welfare achieved under a symmetric history-dependent strategy profile $\boldsymbol{\kappa}^{n}$ is given by

$$
\mathcal{W}_{0}^{n}\left(f_{0}^{n}, s_{0} \mid \boldsymbol{\kappa}^{n}\right)=W_{0}^{n}\left(f_{0}^{n}, s_{0} \mid \boldsymbol{\kappa}^{n}\right)+\mathbb{E}\left\{\sum_{t=1}^{T} W_{t}^{n}\left(f_{t}^{n}, h_{t} \mid \boldsymbol{\kappa}^{n}\right)\right\},
$$

where the expectation is over the future global states $\left\{s_{t}\right\}_{t=1}^{T}$. In particular, we use $\mathcal{W}_{0}^{n}\left(f_{0}^{n}, s_{0} \mid \boldsymbol{\nu}^{n}\right)$ to denote the expected social welfare achieved by the "symmetric dynamic oblivious strategy profile", $\boldsymbol{\nu}^{n}=(\nu, \ldots, \nu)$.

In a continuum model, suppose that all consumers use a common dynamic oblivious strategy $\nu$. Given an initial global state $s_{0}$, the expected social welfare is

$$
\widetilde{\mathcal{W}}_{0}\left(s_{0} \mid \nu\right)=\widetilde{W}_{0}\left(s_{0} \mid \nu\right)+\mathbb{E}\left\{\sum_{t=1}^{T} \widetilde{W}_{t}\left(h_{t} \mid \nu\right)\right\},
$$

where the expectation is over the future global states, $\left\{s_{t}\right\}_{t=1}^{T}$. Here, $\widetilde{W}_{t}\left(h_{t} \mid \nu\right)$ is the stage social welfare under history $h_{t}$ :

$$
\begin{aligned}
\widetilde{W}_{t}\left(h_{t} \mid \nu\right)=-\widetilde{C}\left(\widetilde{A}_{t \mid \nu, h_{t}}, s_{t}\right) & -\widetilde{H}\left(\widetilde{A}_{t-1 \mid \nu, h_{t-1}}, \widetilde{A}_{t \mid \nu, h_{t}}, \bar{s}_{t}\right) \\
& +\sum_{x \in \mathcal{X}_{0}} \eta_{s_{0}}(x) U_{t}\left(l_{\nu, h_{t}}(x), s_{t}, \nu_{t}\left(x, h_{t}\right)\right), \quad t=1, \ldots, T,
\end{aligned}
$$

where $l_{\nu, h_{t}}$ maps a consumer's initial state into her state at stage $t$, under the history $h_{t}$ and the dynamic oblivious strategy $\nu$. The social welfare at stage 0 is given by

$$
\widetilde{W}_{0}\left(s_{0} \mid \nu\right)=-\widetilde{C}\left(\widetilde{A}_{0 \mid \nu, h_{0}}, s_{0}\right)-\widetilde{H}_{0}\left(\widetilde{A}_{0 \mid \nu, h_{0}}, s_{0}\right)+\sum_{x \in \mathcal{X}_{0}} \eta_{s_{0}}(x) U_{0}\left(x, s_{0}, \nu_{0}\left(x, s_{0}\right)\right)
$$

\subsection{Asymptotic social optimality of a DOE}

We now define some notation that will be useful in this subsection. Since there is no idiosyncratic randomness, given a history $h_{t}$, the state of consumer $i$ at stage $t$ depends only on her initial state 
$x_{i, 0}$, and her actions taken at $\tau=0, \ldots, t-1$. At stage $t \geq 1$, the history $h_{t}$ and the transition function $z_{i, t+1}=r\left(x_{i, t}, a_{i, t}, s_{t+1}\right)$ define a mapping $k_{h_{t}}: \mathcal{X}_{0} \times \mathcal{A}^{t} \rightarrow \mathcal{Z}$ :

$$
z_{i, t}=k_{h_{t}}\left(x_{i, 0}, a_{i, 0}, \ldots, a_{i, t-1}\right), \quad t=1, \ldots, T \text {. }
$$

Given an initial state $x_{i, 0}$, consumer $i$ 's total utility under a history $h_{t}$ can be written as a function of her actions taken at stages $\tau=0, \ldots, t$ :

$$
\bar{U}_{h_{t}}\left(x_{i, 0}, a_{i, 0}, \ldots, a_{i, t}\right)=U_{t}\left(x_{i, 0}, s_{0}, a_{i, 0}\right)+\sum_{\tau=1}^{t} U_{t}\left(x_{i, 0}, k_{h_{\tau}}\left(x_{i, 0}, a_{i, 0}, \ldots, a_{i, \tau-1}\right), s_{\tau}, a_{i, \tau}\right) .
$$

Before proving the main result of this section, we introduce a series of assumptions on the convexity and differentiability of the cost and the utility functions.

Assumption 4. We assume the following.

4.1. For any $s \in \mathcal{S}, \widetilde{C}(\cdot, s)$ is convex; for any $\bar{s} \in \mathcal{S}^{2}, \widetilde{H}\left(A, A^{\prime}, \bar{s}\right)$ is convex in $\left(A, A^{\prime}\right)$.

4.2. For any $h_{T} \in \mathcal{H}_{T}$ and any $x_{i, 0} \in \mathcal{X}_{0}$, the function defined in (36) is concave with respect to the vector $\left(a_{i, 0}, \ldots, a_{i, T}\right)$.

4.3. For any $t \geq 1$, any $h_{t} \in \mathcal{H}_{t}$, and any $x_{i, 0} \in \mathcal{X}_{0}$, the function $k_{h_{t}}$ defined in (35) is monotonic in $a_{i, \tau}$, for $\tau=0, \ldots, t-1$; further, its left and right derivatives with respect to $a_{i, \tau}$ exist, for $\tau=0, \ldots, t-1$.

4.4. For $t \geq 1$, and for any $(x, s, a) \in \mathcal{X}_{0} \times \mathcal{S} \times \mathcal{A}$, the left and right derivatives of the utility function $U_{t}(x, z, s, a)$ in $z$ exist.

Assumption 4.1 is standard. If the utility function is concave in $a$, Assumption 4.2 requires that the transition function $k_{h_{t}}$ preserves concavity (a linear function would be an example). Note that Assumptions 4.1 and 4.2 guarantee that in both models (a dynamic game with a finite number of consumers, and the corresponding continuum game), the expected social welfare (consumer $i$ 's expected payoff) is concave in the vector of actions taken by all consumers (respectively, by consumer $i$ ). Assumptions 4.3 and 4.4 ensure the existence of left and right derivatives of the expected social welfare given in (32), with respect to the actions taken by consumers. An example where Assumptions 4.2-4.4 hold is given next. 
ExAmple 2. Consider appliances such as Plug-in Hybrid Electric Vehicles (PHEVs), dish washers, or clothes washers. For such appliances, a customer usually only cares whether a task is completed before a certain time.

Given an initial state (type) of consumer $i, x_{i, 0}$, let $D\left(x_{i, 0}\right)$ and $T\left(x_{i, 0}\right)$ indicate her total desired demand and the stage by which the task has to be completed, respectively. Under a given history $h_{t}$, the total utility accumulated by consumer $i$ until time $t$ is assumed to be of the form

$$
\bar{U}_{h_{t}}\left(x_{i, 0}, a_{i, 0}, \ldots, a_{i, t}\right)=Z\left(x_{i, 0}, \min \left\{D\left(x_{i, 0}\right), \sum_{\tau=0}^{\min \left\{T\left(x_{i, 0}\right), t\right\}} a_{i, \tau}\right\}\right),
$$

for some function $Z$. If for every $x_{i, 0} \in \mathcal{X}_{0}, Z\left(x_{i, 0}, \cdot\right)$ is nondecreasing and concave, then Assumption 4.2 holds. At stage $t=0$, we have

$$
U_{0}\left(x_{i, 0}, s_{0}, a_{i, 0}\right)=Z\left(x_{i, 0}, \min \left\{D\left(x_{i, 0}\right), a_{i, 0}\right\}\right) .
$$

For $t=1, \ldots, T\left(x_{i, 0}\right)$, we let $z_{i, t}=\sum_{\tau=0}^{t-1} a_{i, \tau}$, and

$$
U_{t}\left(x_{i, 0}, z_{i, t}, s_{t}, a_{i, t}\right)=Z\left(x_{i, 0}, \min \left\{D\left(x_{i, 0}\right), a_{i, t}+z_{i, t}\right\}\right)-Z\left(x_{i, 0}, \min \left\{D\left(x_{i, 0}\right), z_{i, t}\right\}\right) .
$$

For $t \geq T\left(x_{i, 0}\right)+1$, we let $z_{i, t}=D\left(x_{i, 0}\right)$, and let $U_{t}\left(x_{i, t}, s_{t}, a_{i, t}\right)$ be identically zero. Suppose that for every $x_{i, 0} \in \mathcal{X}_{0}$, the right and left derivatives of $Z\left(x_{i, 0}, \cdot\right)$ exist. Then, Assumptions 4.3 and 4.4 hold.

Theorem 2. Suppose that Assumptions 2-4 hold. Let $\nu$ be a DOE of the continuum game. Then, the following hold.

(a) In the continuum game, the social welfare is maximized (over all symmetric dynamic oblivious strategy profiles) at the DOE, i.e., ${ }^{9}$

$$
\widetilde{\mathcal{W}}_{0}\left(s_{0} \mid \nu\right)=\sup _{\vartheta \in \mathfrak{V}} \widetilde{\mathcal{W}}_{0}\left(s_{0} \mid \vartheta\right), \forall s_{0} \in \mathcal{S}
$$

where $\mathfrak{V}$ is the set of all dynamic oblivious strategies.

\footnotetext{
${ }^{9}$ Note that we are only comparing the social welfare under different symmetric dynamic oblivious strategy profiles, where all consumers are using the same dynamic oblivious strategy $(\nu$ or $\vartheta$ ). This is no loss of generality because under Assumption 4, the social welfare in a continuum game is a concave function of the collection of consumer actions taken under the different histories. Hence, it can be shown that the optimal social welfare can be achieved by a symmetric dynamic oblivious strategy profile.
} 
(b) For a sequence of $n$-consumer games, the symmetric DOE strategy profile, $\boldsymbol{\nu}^{n}=(\nu, \ldots, \nu)$, approximately maximizes the expected social welfare, as the number of consumers increases to infinity. That is, for any initial global state $s_{0}$, and any sequence of symmetric history-dependent strategy profiles $\left\{\boldsymbol{\kappa}^{n}\right\}$, we have $e^{10}$

$$
\limsup _{n \rightarrow \infty} \mathbb{E}\left\{\frac{\mathcal{W}_{0}^{n}\left(f_{0}^{n}, s_{0} \mid \boldsymbol{\kappa}^{n}\right)-\mathcal{W}_{0}^{n}\left(f_{0}^{n}, s_{0} \mid \boldsymbol{\nu}^{n}\right)}{n}\right\} \leq 0
$$

where the expectation is over the initial population state, $f_{0}^{n}$.

The proof of Theorem 2 is given in Appendix D.

\section{Conclusion and Future Directions}

In an electric power system, load swings may result in significant ancillary cost to suppliers. Motivated by the observation that marginal cost pricing may not achieve social optimality in electricity markets, we proposed a new dynamic pricing mechanism that takes into account the externality conferred by a consumer's action on future ancillary cost. Besides proposing a suitable gametheoretic model that incorporates the cost of load fluctuations and a particular pricing mechanism for electricity markets, a main contribution of this paper was to show that the proposed pricing mechanism achieves social optimality in a dynamic nonatomic game, and approximate social optimality for the case of finitely many consumers, under certain convexity assumptions.

To compare the proposed pricing mechanism with marginal cost pricing, we presented a numerical example in which the demand increases sharply at the last stage. In this example, the proposed pricing mechanism creates a stronger incentive for consumers to shift their peak load than marginal cost pricing, through an additional negative price charged on off-peak consumer demand. As a result, compared with marginal cost pricing, the proposed pricing mechanism achieves a higher social welfare, and at the same time, reduces the peak load, and therefore has the potential to reduce the need for long-term investments in peaking plants.

\footnotetext{
${ }^{10}$ Under Assumption 4, the social welfare in an $n$-consumer game is a concave function of the collection of consumer actions taken under the different histories. Therefore, $\sup _{\kappa^{n} \in \mathfrak{K}_{n}} \mathcal{W}_{0}^{n}\left(f_{0}^{n}, s_{0} \mid \boldsymbol{\kappa}^{n}\right)$ is also the maximum social welfare that can be achieved by a (possibly non-symmetric) history-dependent strategy profile.
} 
We believe that the constructed dynamic game-theoretic model, the proposed pricing mechanism, and more importantly, the insights provided by this work, can be applied to a more general class of markets with friction. As an extension and future work, one can potentially develop and use variations of our framework to a market of a perishable product/service where demand fluctuations incur significant cost to suppliers. Examples include data centers implementing cloud services that suffer from the switching costs to toggle a server into and out of a power-saving mode (Lin et al. 2011), and large organizations such as hospitals that use on-call staff to meet unexpected demand.

\section{References}

Al-Najjar N. 2004. Aggregation and the law of large numbers in large economies, Games and Economic Behavior, 47, 1-35.

Al-Najjar N. 2008. Large games and the law of large numbers, Games and Economic Behavior, 64, 1-34.

Adlakha S., R. Johari, G. Y. Weintraub, A. Goldsmith. 2011. Equilibria of dynamic games with many players: existence, approximation, and market structure, Working paper, Stanford Univerisity. http: //www.arxiv.org/abs/1011.5537

Barbose G., R. Wiser, A. Phadke, C. Goldman. 2008. Reading the tea leaves: How utilities in the West are managing carbon regulatory risk in their resource plan, Lawrence Berkeley National Laboratory, Tech. Rep. LBNL-44E, March 2008.

Bergin J., D. Bernhardt. 1992. Anonymous sequential games with aggregate uncertainty, Journal of Mathematical Economics, 21(6), 543-562.

Bodoh-Creed A. 2010. Mean field approximation of large games, Working paper, Cornell University.

Bodoh-Creed A. 2011. Approximation of large games with applications to uniform price auctions, Working paper, Cornell University. http://www.arts.cornell.edu/econ/ab882/LargeGames.pdf

Borenstein S., M. Jaske, A. Rosenfeld. 2002. Dynamic pricing, advanced metering and demand response in electricity markets, http://escholarship.org/uc/item/11w8d6m4

Chao H. 2010. Price-responsive demand management for a smart grid world, The Electricity Journal, 23(1), $7-20$.

Cho I., S. P. Meyn. 2010a. Efficiency and marginal cost pricing in dynamic competitive markets with friction, Theoretical Economics, 5(2), 215-239. 
Cho I., S. P. Meyn. 2010b. A dynamic newsboy model for optimal reserve management in electricity markets, Working paper, University of Illinois at Urbana-Champaign. https://netfiles.uiuc.edu/meyn/www/ spm_files/Papers_pdf/newsboy2010-8-23.pdf

Doraszelski, U., A. Pakes. 2007. A framework for applied dynamic analysis in IO. Handbook of Industrial Organization, Vol. 3.

Fudenberg, D., J. Tirole. 1991. Game Theory, The MIT Press.

Fudenberg D., D. K. Levine 1993. Self-confirming equilibrium, Econometrica, 61(3), 523-545.

Hoeffding W. 1963. Probability inequalities for sums of bounded random variables, Journal of the American Statistical Association, 58(301), 13-30.

Hogan. W. 2010. Demand response pricing in organized wholesale markets, IRC Comments, Demand Reponse Notice of Proposed Rulemaking, FERC Docket RM10-17-000. http://www.hks.harvard.edu/fs/ whogan/Hogan_IRC_DR_051310.pdf

ISO New England Inc. 2010. 2009 Annual markets report. http://www.iso-ne.com/markets/mktmonmit/ rpts/other/amr09_final_051810.pdf

ISO New England Inc. 2011. Daily Report. www.ferc.gov/market-oversight/mkt-electric/ new-england/2011/02-2011-elec-isone-dly.pdf

Kalai E., E. Lehrer 1995. Subjective games and equilibria, Games and Economic Behavior, 8(1), 123-163.

Kizilkale A. C., S. Mannor (2010), Regulation and efficiency in markets with friction, Proceedings of the Forty-Eighth Annual Allerton Conference, Monticello, IL, USA.

Lin M., A. Wierman, L. Andrew, E. Thereska. 2011. Dynamic right-sizing for power-proportional data centers, Proceedings of IEEE INFOCOM, Shanghai, China.

Lucas R. E., E. C. Prescott. 1971. Investment under uncertianty, Econometrica, 39(5), 659-681.

Mansur E. 2008. Measuring welfare in restructured electricity markets, The Review of Economics and Statistics, 90(2), 369-386.

Mas-Colell, A., X. Vives. 1993. Implementation in economies with a continuum of agents, The Review of Economic Studies, 60(3), 613-629.

Maskin, E., J. Tirole. 1988. A theory of dynamic oligopoly, I and II, Econometrica, 56(3), 549-570. 
Milchtaich I. 2004. Social optimality and cooperation in nonatomic congestion games, Journal of Economic Theory, 141(1), 56-87.

Muth J. F. 1961. Rational expectations and the theory of price movements, Econometrica, 29(3), 315-335.

Roughgarden T., E. Tardos. 2004. Bounding the inefficiency of equilibria in nonatomic congestion games, Games and Economic Behavior, 47(2), 389-403.

Roozbehani M., M. Dahleh, S. Mitter. 2010. On the stability of wholesale electricity markets under real-time pricing, Proceedings of the 49th IEEE Conference on Decision and Control, Atlanta, GA, USA.

Spees K., L. Lave. 2008. Impacts of responsive load in PJM: load shifting and real time pricing, The Energy Journal, 29(2), 101-122.

Stoft S. 2002. Power system economics: designing markets for electricity, IEEE Press.

US Department of Energy. 2006. Benefits of demand response in electricity markets and recommendations for achieving them: a report to the United States Congress pursuant to Section 1252 of the energy policy act of 2005. http://eetd.1bl.gov/ea/ems/reports/congress-1252d.pdf

Weintraub, G. Y., C. L. Benkard, B. Van Roy. 2008. Markov perfect industry dynamics with many firms, Econometrica, 76(6), 1375-1411.

Weintraub G., C. Benkard, B. Van Roy. 2010. Computational Methods for Oblivious Equilibrium, Operations Research, 58(4), 1247-1265.

\section{Acknowledgments}

The authors are grateful to Prof. Michael Caramanis for discussions and several comments on a draft of this paper, and to Prof. Ramesh Johari for discussions and pointers to the literature. This research was supported in part by the National Science Foundation under grant CMMI-0856063 and by a Graduate Fellowship from Shell. 


\section{Electronic Companion}

\section{A. Approximation of the supplier cost}

In this appendix, we show via simulation that at least in some cases, the supplier cost (including the cost of ancillary service) can be captured by a simplified cost function of the form in (5). We consider a $(T+1)$-stage dynamic model with two energy resources, a primary energy resource and an ancillary energy resource. It is assumed that the forecast demand is met by the primary energy resource (e.g., coal-fired or nuclear power generators), and that at stage $t=1, \ldots, T$, the deviations from the forecast demand, $\left\{w_{t}\right\}_{t=1}^{T}$, are independent random variables uniformly distributed on $[-\omega, \omega]$. At the initial stage 0 , we assume that the forecast error is zero, i.e., $w_{0}=0$.

At stage $t$, let $b_{t}$ denote the difference between the actual output of the primary energy resource and the forecast demand, and let $d_{t}$ denote the output of the ancillary energy resource (e.g., oil/gas combustion turbines). For simplicity, we will assume that the cost of a positive primary energy resource (respectively, ancillary energy resource) is $b_{t}^{2}$ (respectively, $10 d_{t}^{2}$ ).

Let $r_{b}$ be the ramping rate of the primary energy resource, and $r_{d}$ be the ramping rate of the ancillary energy resource. At the initial stage 0 , we assume that $b_{0}=w_{0}=0$, and $d_{0}=0$. At stage $t \geq 1$, if $w_{t}<0$, then $d_{t}=0$, and we assume that $b_{t}=0$, that is, the system operator maintains a high level of (potential) output in order to be able to deal with a possible unexpected demand surge in the future; if $w_{t}>0$, we assume that $b_{t}=\min \left\{w_{t}, b_{t-1}+r_{b}\right\}$, where $b_{t-1}+r_{b}$ is the maximum possible output of the primary energy resource at stage $t$, and that $d_{t}=\min \left\{w_{t}-b_{t}, d_{t-1}+r_{d}\right\}$. The total supplier cost (excluding the cost to meet the forecast demand) is

$$
C=\sum_{t=1}^{T}\left(b_{t}^{2}+10 d_{t}^{2}\right) .
$$

For notational convenience, we let $(\cdot)^{+}=\max \{\cdot, 0\}$. We use the following function to approximate the supplier cost:

$$
\widetilde{C}=\sum_{t=1}^{T}\left(\widetilde{b}_{t}^{2}+10{\widetilde{d_{t}^{2}}}^{2},\right.
$$

where $\widetilde{d}_{t}=\min \left\{r_{d},\left(0, w_{t}-\left(w_{t-1}\right)^{+}-r_{b}\right)^{+}\right\}$, and $\widetilde{b}_{t}=\left(w_{t}-\widetilde{d}_{t}\right)^{+}$. 


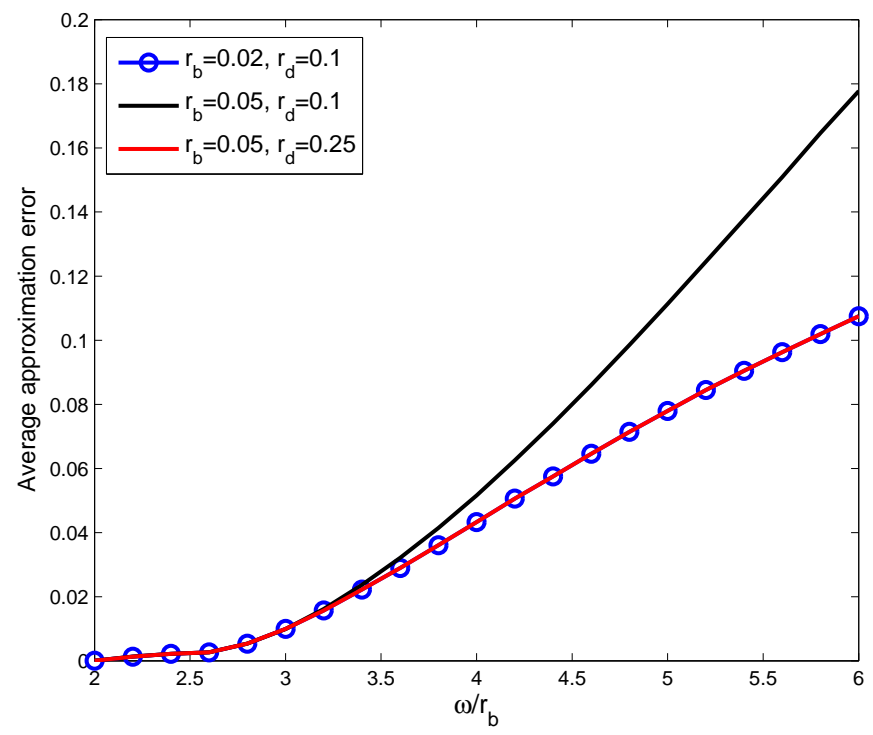

Figure 2 A simulation experiment with $T=24$, and 500,000 trajectories for each $\omega / r_{b}$ on the horizontal axis. The approximation error is defined by $|C-\widetilde{C}| / C$. The average approximation error (vertical axis) is the mean value of the approximation errors of the 500,000 trajectories.

The function in (38) well approximates the supplier cost in (37), if for an unexpected demand surge at stage $t$, the system load at the previous stage, $w_{t-1}$, is met by the primary energy resource, and load shedding rarely occurs (so that $\left(w_{t}\right)^{+}$typically equals $b_{t}+d_{t}$ ). Note that in (38), for each stage $t$, the approximated cost depends only on $w_{t-1}$ and $w_{t}$. Therefore, the approximated cost in (38) can be written as

$$
\widetilde{C}=\sum_{t=1}^{T}\left(\left(\left(w_{t}\right)^{+}\right)^{2}+H\left(w_{t-1}, w_{t}\right)\right),
$$

where $H\left(w_{t-1}, w_{t}\right)=\left(\widetilde{b}_{t}^{2}+10 \widetilde{d}_{t}^{2}-\left(\left(w_{t}\right)^{+}\right)^{2}\right)^{+}$.

For different values of the parameters, $r_{b}, r_{d}$, and $\omega$, we evaluate the performance of the approximation via simulation. Fig. 2 depicts some numerical results of a simulation experiment and we can make the following observations:

1. The main source of approximation error is from the following scenario: at stage $t-1$, the deviation in demand $w_{t-1}$ is nonpositive, $w_{t}>r_{b}$, and $w_{t+1}>2 r_{b}$. In this scenario, the output of the primary energy source at stage $t$ is $r_{b}$, which is less than $w_{t}$. When $\omega / r_{b} \leq 2$, this scenario never occurs and we observe from Fig. 2 that the approximation error is close to zero, regardless of the 
value of $r_{d}$.

2. Comparing the black curve with the red curve in Fig. 2, we observe that when $\omega / r_{b}>3$ (when $r_{b}=0.05$ and $\left.\omega>0.15\right)$, the approximation error for the case where $r_{d}=0.1$ is larger than that for the case where $r_{d}=0.25$. This is because for the case with $r_{d}=0.1$, as $\omega / r_{b}$ increases from 3 to 6 (as $\omega$ increases from 0.15 to 0.3 ), the probability of load shedding increases, which deteriorates the performance of the approximation.

3. Finally, and perhaps most importantly, when the ramping rate of the ancillary energy resource is high enough to prevent any load shedding, the approximation error is an increasing function of the single parameter $\omega / r_{b}$ (e.g., the blue curve with circle markers for $r_{b}=0.02, r_{d}=0.1$ and the red curve for $r_{b}=0.05, r_{d}=0.25$ merge together in Fig. 2); in this case, we observe from Fig. 2 that the approximation error is less than $10 \%$ for a wide range of parameter values.

\section{B. Implementation of the proposed pricing mechanism}

To implement a dynamic real-time pricing mechanism, all consumers should be exposed to timevarying prices associated with ex ante estimates of generation costs that reflect system operating conditions (p. 81 of Borenstein et al. (2002)) so that they can adjust their demand in accordance to real-time prices as well as ex ante price estimates. The mechanism proposed in this paper is not an exception. The ex ante estimates of real-time prices can be developed by evaluating statistical relationships between historical real-time prices and various factors such as load forecast, weather predictions, and expected supply/demand balances (Borenstein et al. 2002).

We now provide a brief discussion of the details of a possible implementation of the proposed pricing mechanism:

Ex ante price estimates. Suppose that the exogenous state $s_{t}$ is realized at the beginning of each stage $t$; for every possible realization of the trajectory (scenario) of future exogenous states $\left\{s_{\tau}\right\}_{\tau=t+1}^{t+T}$, consumers receive corresponding price estimates $\left\{\hat{p}_{\tau}\right\}_{\tau=t}^{t+T},\left\{\hat{w}_{\tau}\right\}_{\tau=t}^{t+T}$, and $\left\{\hat{q}_{\tau}\right\}_{\tau=t}^{t+T}$, from utilities and/or the independent system operator. The consumers also know or receive the probabilities of the different trajectories. With the received price estimates (associated with possible 
trajectories of future exogenous states) and preset utility functions, each consumer's infrastructure solves a dynamic programming problem to maximize her expected payoff over the horizon from $t$ to $t+T$. (The state at time $\tau$ in this dynamic program is comprised of $y_{i, \tau}$, and the history $\left(s_{t}, s_{t+1}, \ldots, s_{\tau}\right.$. $)$ The dimension of this state space grows with the time horizon $T$ (because of the exponentially increasing number of histories). Unfortunately, this is unavoidable for models of this type, and might require some further approximations, e.g., in the spirit of Weintraub et al. (2010). Ex post prices. At each stage $t$, after the realization of the system demands $A_{t-1}$ and $A_{t}$, consumers pay ex post prices $\left(p_{t}, w_{t}, q_{t}\right)$ that are determined according to Eqs. (11) and (12).

Equilibrium. In a market with a large number of price-taking consumers, it is possible to make ex ante price estimates (contingent on the realized trajectories) that are close to ex post prices. If every consumer maximizes her own payoff in response to these pretty accurate price estimates, the resulting outcome should be close to that resulting from a Rational Expectations Equilibrium (REE). The results derived in this paper show that the expected social welfare can be approximately maximized, under the proposed mechanism.

We emphasize here that there remain several challenging implementation issues, e.g., the accuracy of future price estimates and the uncertainty of consumer response to ex ante price estimates. For example, Roozbehani et al. (2010) show that if consumers act myopically to highly inaccurate price estimates, real-time pricing may result in extreme price volatility. However, we note that these challenges are generic to almost all kinds of real-time pricing mechanisms.

\section{Proof of Theorem 1}

We consider a sequence of $n$-consumer models where $n-1$ consumers (all except for consumer i) use a DOE strategy $\nu$. As the number of consumers increases to infinity, the randomness of consumer initial states averages out. Thus, in Step 1 we show that the aggregate demand (in an $n$-consumer model) at a history $h_{t}$ is close to $n \widetilde{A}_{t \mid \nu, h_{t}}$ (defined in Eq. (16)), with high probability. As a consequence, we show in Step 2 that as $n \rightarrow \infty$, consumer $i$ 's expected payoff associated with any sequence of actions can be approximated by her oblivious value defined in (21). Since the DOE 
strategy $\nu$ maximizes consumer $i$ 's oblivious value among all possible strategies, we argue in Step 3 that as $n \rightarrow \infty$, the maximum expected payoff consumer $i$ can obtain is asymptotically no larger than the optimal oblivious value. In Step 4, we show that consumer $i$ 's optimal oblivious value can be approximately achieved if she uses the DOE strategy $\nu$. We finally conclude with the AME property of the DOE strategy $\nu$ (the result in Theorem 1).

In what follows, we will be using the uniform metric over the set of probability distributions on the finite set $\mathcal{X}_{0}$. Specifically, if $f$ and $f^{\prime}$ are two distributions on $\mathcal{X}_{0}$, we let

$$
d\left(f, f^{\prime}\right) \triangleq\left\|f-f^{\prime}\right\|_{\infty}=\max _{x \in \mathcal{X}_{0}}\left|f(x)-f^{\prime}(x)\right|
$$

Step 1: With high probability, the aggregate demand under a history $h_{t}$ is close to $n \widetilde{A}_{t \mid \nu, h_{t}}$.

Given an initial distribution $f_{-i, 0}^{n}$, and if all consumers (excluding $i$ ) use a dynamic oblivious strategy $\nu$, we write their aggregate demand at a history $h_{t}$ as

$$
A_{-i, t}^{n}=(n-1) \sum_{x \in \mathcal{X}_{0}} f_{-i, 0}^{n}(x) \nu_{t}\left(x, h_{t}\right)
$$

Recall that (cf. (16))

$$
\widetilde{A}_{t \mid \nu, h_{t}}=\sum_{x \in \mathcal{X}_{0}} \eta_{s_{0}}(x) \cdot \nu_{t}\left(x, h_{t}\right) .
$$

We observe that if $d\left(f_{-i, 0}^{n}, \eta_{s_{0}}\right) \leq \delta /(X B)$, then at any history $h_{t}$ we have

$$
\left|A_{-i, t}^{n}-(n-1) \widetilde{A}_{t \mid \nu, h_{t}}\right| \leq \delta(n-1)
$$

with probability at least $1-O\left(e^{-n}\right)$. More precisely, since the consumers' initial states are independently drawn according to $\eta_{s_{0}}$, Hoeffding's inequality (Hoeffding (1963)) yields,

$$
\mathbb{P}\left(d\left(F_{s_{0}}^{n-1}, \eta_{s_{0}}\right) \geq \delta /(X B)\right) \leq 2 X \exp \left\{-2(n-1) \delta^{2} /\left(X^{2} B^{2}\right)\right\}, \forall s_{0} \in \mathcal{S}, \forall \delta>0, \forall n \in \mathbb{N}^{+},
$$

where $X$ is the cardinality of the set $\mathcal{X}_{0}$ and $F_{s_{0}}^{n-1}$ is an $X$-dimensional random vector denoting the distribution of the initial states of the $n-1$ consumers (excluding $i$ ).

Step 2: Under a given history $h_{T}$, consumer $i$ 's expected payoff can be approximated by a corresponding oblivious value, defined in (45). 
In an $n$-consumer model, suppose that all consumers other than $i$ use a dynamic oblivious strategy $\nu$. Given a complete history $h_{T}=\left(s_{0}, \ldots, s_{T}\right)$, and consumer $i$ 's initial state $x_{i, 0}$, we define her expected payoff under a history-dependent strategy $\kappa^{n}$ by

$$
V_{i, 0}^{n}\left(x_{i, 0}, h_{T} \mid \kappa^{n}, \nu\right)=\mathbb{E}\left\{V_{i, 0}^{n}\left(x_{i, 0}, h_{T}, f_{-i, 0}^{n} \mid \kappa^{n}, \nu\right)\right\}
$$

where the expectation is over the initial distribution, $f_{-i, 0}^{n}$, and $V_{i, 0}^{n}\left(x_{i, 0}, h_{T}, f_{-i, 0}^{n} \mid \kappa^{n}, \nu\right)$ is consumer $i$ 's payoff under the given initial distribution $f_{-i, 0}^{n}$,

$$
V_{i, 0}^{n}\left(x_{i, 0}, h_{T}, f_{-i, 0}^{n} \mid \kappa^{n}, \nu\right)=\sum_{t=0}^{T} \pi_{i, t}^{n}\left(y_{i, t}, h_{t}, f_{-i, t}^{n} \mid \kappa^{n}, \nu\right),
$$

and where the stage payoff function, $\pi_{i, t}^{n}(\cdot)$, has been defined in $(23)$. Note that given $f_{-i, 0}^{n}$, and since all consumers other than $i$ use a dynamic oblivious strategy, the distribution of their augmented states, $f_{-i, t}^{n}$, is completely determined by the history $h_{t}$. Therefore, given $f_{-i, 0}^{n}$, consumer $i$ 's historydependent strategy $\kappa^{n}$ is equivalent to a dynamic oblivious strategy: the action it takes at stage $t$ depends only on $x_{i, 0}$ and $h_{t}$. We can therefore define an oblivious strategy $\widetilde{\nu}^{n}\left(\kappa^{n}, f_{-i, 0}^{n}\right)$ such that

$$
\widetilde{\nu}_{t}\left(\kappa^{n}, f_{-i, 0}^{n}\right)\left(x_{i, 0}, h_{t}\right)=\kappa_{t}^{n}\left(y_{i, t}, h_{t}, f_{-i, t}^{n}\right)
$$

where $f_{-i, t}^{n}$ is the distribution of the $n-1$ consumers' augmented states under the history $h_{t}$, induced from the initial distribution $f_{-i, 0}^{n}$ by the symmetric oblivious strategy profile $(\nu, \ldots, \nu)$, and $y_{i, t}$ is consumer $i$ 's augmented state under the history $h_{t}$, induced from her initial state $x_{i, 0}$ by the strategy $\kappa^{n}$.

In the corresponding continuum model, suppose that all consumers other than $i$ use a dynamic oblivious strategy $\nu$. For a given complete history $h_{T}$, we define consumer $i$ 's oblivious value under an initial distribution $f_{-i, 0}^{n}$, her initial state $x_{i, 0}$, and the history-dependent strategy $\kappa^{n}$ :

$$
\widetilde{V}_{i, 0}\left(x_{i, 0}, h_{T}, f_{-i, 0}^{n} \mid \kappa^{n}, \nu\right)=\sum_{t=0}^{T} \widetilde{\pi}_{i, t}\left(y_{i, t}, h_{t} \mid \widetilde{\nu}\left(\kappa^{n}, f_{-i, 0}^{n}\right), \nu\right),
$$

where the oblivious stage value function $\widetilde{\pi}_{i, t}(\cdot)$ is given in $(20)$. We define the expected oblivious value for consumer $i$ under the history-dependent strategy $\kappa^{n}$, as ${ }^{11}$

\footnotetext{
${ }^{11}$ This is actually the oblivious value achieved by a mixed strategy under the complete history $h_{T}$. In the continuum model, under a history $h_{t}$, the mixed strategy takes an action $\widetilde{\nu}_{t}\left(\kappa^{n}, f_{-i, 0}^{n}\right)\left(x_{i, 0}, h_{t}\right)$, if the distribution of the $n-1$ consumers' (excluding $i$ 's) initial states in the corresponding $n$-consumer model is realized as $f_{-i, 0}^{n}$.
} 


$$
\widetilde{V}_{i, 0}\left(x_{i, 0}, h_{T} \mid \kappa^{n}, \nu\right)=\mathbb{E}\left\{\widetilde{V}_{i, 0}\left(x_{i, 0}, h_{T}, f_{-i, 0}^{n} \mid \kappa^{n}, \nu\right)\right\}
$$

where the expectation is over the initial distribution, $f_{-i, 0}^{n}$. For any $\varepsilon>0$, in this step we aim to show that there exists a positive integer $N$ such that for any sequence of history-dependent strategies $\left\{\kappa^{n}\right\}$,

$$
\left|\widetilde{V}_{i, 0}\left(x_{i, 0}, h_{T} \mid \kappa^{n}, \nu\right)-V_{i, 0}^{n}\left(x_{i, 0}, h_{T} \mid \kappa^{n}, \nu\right)\right| \leq \varepsilon, \quad \forall n \geq N, \quad \forall h_{T} \in \mathcal{H}_{T}, \quad \forall x_{i, 0} \in \mathcal{X}_{0}
$$

For a given $s_{0}$, let $\mathfrak{F}_{s_{0}}^{n-1}(\delta)$ be the set of $f_{-i, 0}^{n}$ such that $d\left(f_{-i, 0}^{n}, \eta_{s_{0}}\right) \leq \delta$. To verify (46), we first argue that for any $\varepsilon>0$, there exists an positive integer $N_{1}$ and some $\delta>0$ such that for any $f_{-i, 0}^{n} \in \mathfrak{F}_{s_{0}}^{n-1}(\delta /(X B))$ and any $n \geq N_{1}$,

$$
\left|\widetilde{V}_{i, 0}\left(x_{i, 0}, h_{T}, f_{-i, 0}^{n} \mid \kappa^{n}, \nu\right)-V_{i, 0}^{n}\left(x_{i, 0}, h_{T}, f_{-i, 0}^{n} \mid \kappa^{n}, \nu\right)\right| \leq \varepsilon / 2, \quad \forall h_{T} \in \mathcal{H}_{T}, \quad \forall x_{i, 0} \in \mathcal{X}_{0}
$$

Under the uniform equicontinuity assumption for the derivatives of the cost functions (see Eqs. (25) and (26)), we know that a small deviation of the aggregate demand from $\widetilde{A}_{t \mid \nu, h_{t}}$ will result in prices that are only slightly different from the prices in the continuum model. We also note that consumer $i$ cannot take an action larger than $B$, and her payoff is influenced by other consumers only through the prices. For any $\varepsilon>0$, we can find some $\delta>0$ and a positive integer $N_{1}$ such that for any given $\left(x_{i, 0}, h_{t}\right)$, if $f_{-i, 0}^{n} \in \mathfrak{F}_{s_{0}}^{n-1}(\delta /(X B))$, then the inequality in (41) holds for any history $h_{\tau}$, which implies that

$$
\left|\tilde{\pi}_{i, t}\left(y_{i, t}, h_{t}, \kappa_{t}^{n}\left(y_{i, t}, h_{t}, f_{-i, t}^{n}\right) \mid \nu\right)-\pi_{i, t}^{n}\left(y_{i, t}, h_{t}, f_{-i, t}^{n} \mid \kappa^{n}, \nu\right)\right| \leq \varepsilon /(2 T+2), \forall n \geq N_{1}, \forall h_{t},
$$

i.e., consumer $i$ 's stage payoff (under the action $\kappa_{t}^{n}\left(y_{i, t}, h_{t}, f_{-i, t}^{n}\right)$ ) in the $n$-consumer model is close to her oblivious stage value (under the same action $\left.\kappa_{t}^{n}\left(y_{i, t}, h_{t}, f_{-i, t}^{n}\right)\right)$ in the continuum model, if the initial distribution in the $n$-consumer model, $f_{-i, 0}^{n}$, is close to its expectation. The result in (47) follows from Eq. (48) and the definitions in (43) and (44). Note that $Q+2 B P$ is an upper bound on the stage payoff that consumer $i$ could obtain, and $-2 B P$ is a lower bound on consumer $i$ 's stage payoff, under Assumption 3. The desired result in (46) follows from (47), and the fact that the probability that $f_{-i, 0}^{n} \notin \mathfrak{F}_{s_{0}}^{n-1}(\delta /(X B))$ decays exponentially with $n$ (cf. Eq. (42)). 
Step 3: The maximum expected payoff consumer $i$ can obtain is asymptotically no larger than the optimal oblivious value.

In this step, we consider the case where all consumers in an $n$-consumer model except for $i$ use a DOE strategy $\nu$, and argue that for any sequence of history-dependent strategies $\left\{\kappa^{n}\right\}$,

$$
\limsup _{n \rightarrow \infty}\left(V_{i, 0}^{n}\left(x_{i, 0}, s_{0} \mid \kappa^{n}, \nu\right)-\widetilde{V}_{i, 0}\left(x_{i, 0}, s_{0} \mid \nu, \nu\right)\right) \leq 0, \quad \forall s_{0} \in \mathcal{S}, \quad \forall x_{i, 0} \in \mathcal{X}_{0}
$$

where consumer $i$ 's expected payoff, $V_{i, 0}^{n}\left(x, s \mid \kappa^{n}, \nu\right)$, is given in $(24)$, and $\widetilde{V}_{i, 0}(x, s \mid \nu, \nu)$ is the oblivious value function in (21). We first observe that

$$
V_{i, 0}^{n}\left(x_{i, 0}, s_{0} \mid \kappa^{n}, \nu\right)=\sum_{h_{T} \in \mathcal{H}_{T}\left(s_{0}\right)} \mathbb{P}\left(h_{T} \mid s_{0}\right) \cdot V_{i, 0}^{n}\left(x_{i, 0}, h_{T} \mid \kappa^{n}, \nu\right),
$$

where $\mathcal{H}_{T}\left(s_{0}\right)$ is the set of complete histories commencing at state $s_{0}$, and $\mathbb{P}\left(h_{T} \mid s_{0}\right)$ is the probability that the history $h_{T}$ is realized, conditional on the initial global state being $s_{0}$. We define

$$
\widetilde{V}_{i, 0}\left(x_{i, 0}, s_{0} \mid \kappa^{n}, \nu\right)=\sum_{h_{T} \in \mathcal{H}_{T}\left(s_{0}\right)} \mathbb{P}\left(h_{T} \mid s_{0}\right) \cdot \widetilde{V}_{i, 0}\left(x_{i, 0}, h_{T} \mid \kappa^{n}, \nu\right)
$$

Note that if $\kappa^{n}$ happens to be a dynamic oblivious strategy, this definition is consistent with the definition of oblivious value function in (21).

For any $\varepsilon>0$, let $N$ be the integer defined in Eq. (46); for any sequence of history-dependent strategies $\left\{\kappa^{n}\right\}$, we argue that

$$
\begin{aligned}
\widetilde{V}_{i, 0}\left(x_{i, 0}, s_{0} \mid \nu, \nu\right) & \geq \sum_{h_{T} \in \mathcal{H}_{T}\left(s_{0}\right)} \mathbb{P}\left(h_{T} \mid s_{0}\right) \cdot \widetilde{V}_{i, 0}\left(x_{i, 0}, h_{T} \mid \kappa^{n}, \nu\right) \\
& \geq \sum_{h_{T} \in \mathcal{H}_{T}\left(s_{0}\right)} \mathbb{P}\left(h_{T} \mid s_{0}\right) \cdot\left(V_{i, 0}^{n}\left(x_{i, 0}, h_{T} \mid \kappa^{n}, \nu\right)-\varepsilon\right) \\
& =V_{i, 0}^{n}\left(x_{i, 0}, s_{0} \mid \kappa^{n}, \nu\right)-\varepsilon, \quad \forall n \geq N, \quad \forall x_{i, 0} \in \mathcal{X}_{0} .
\end{aligned}
$$

The DOE strategy $\nu$, by definition, maximizes consumer $i$ 's oblivious value function among all possible dynamic oblivious strategies. The first inequality in (52) follows from the fact that $\widetilde{V}_{i, 0}\left(x_{i, 0}, s_{0} \mid\right.$ $\left.\kappa^{n}, \nu\right)$ is a weighted sum of the oblivious values achieved by a family of dynamic oblivious strategies $^{12}$. The second inequality in (52) is due to (46), and the last equality in (52) follows from (50). The desired result, (49), follows.

\footnotetext{
${ }^{12}$ Note that for a given $f_{-i, 0}^{n}$, the action taken by $\kappa^{n}$ depends only on $x_{i, 0}$ and $h_{t}$, and that $\widetilde{V}_{i, 0}\left(x_{i, 0}, h_{T} \mid \kappa^{n}, \nu\right)$ is the oblivious value achieved by a mixed strategy; cf. the footnote associated with (45).
} 
Step 4: Consumer i's optimal oblivious value can be asymptotically achieved at an $n$-consumer game under a DOE strategy.

In this step, we consider the case where all consumers in an $n$-consumer model use a DOE strategy $\nu$, and show that

$$
\lim _{n \rightarrow \infty}\left(\widetilde{V}_{i, 0}\left(x_{i, 0}, s_{0} \mid \nu, \nu\right)-V_{i, 0}^{n}\left(x_{i, 0}, s_{0}, \mid \nu, \nu\right)\right)=0, \quad \forall s_{0} \in \mathcal{S}, \quad \forall x_{i, 0} \in \mathcal{X}_{0}
$$

According to (46), with $\kappa^{n}=\nu$, for any $\varepsilon>0$, we can find some $N$ such that

$$
\left|\widetilde{V}_{i, 0}\left(x_{i, 0}, h_{T} \mid \nu, \nu\right)-V_{i, 0}^{n}\left(x_{i, 0}, h_{T} \mid \nu, \nu\right)\right| \leq \varepsilon, \quad \forall n \geq N, \quad \forall h_{T} \in \mathcal{H}_{T}, \quad \forall x_{i, 0} \in \mathcal{X}_{0}
$$

The desired result in (53) follows from (50) and (51). Theorem 1 follows from (49) and (53).

\section{Proof of Theorem 2}

\section{D.1. Proof of Part (a)}

We will show that in a continuum model, a DOE strategy maximizes the expected social welfare among all possible symmetric dynamic oblivious strategy profiles (part (a) of the theorem), i.e., that if $\nu$ is DOE, then

$$
\widetilde{\mathcal{W}}_{0}\left(s_{0} \mid \nu\right)=\sup _{\vartheta \in \mathfrak{V}} \widetilde{\mathcal{W}}_{0}\left(s_{0} \mid \vartheta\right), \quad \forall s_{0} \in \mathcal{S}
$$

Let $S$ and $X$ be the cardinality of $\mathcal{S}$ and $\mathcal{X}_{0}$, respectively. Given the initial global state $s_{0}$, the number of possible histories of length $t+1$ is $S^{t}$. Hence, the number of all possible histories commencing at state $s_{0}$ is $\sum_{t=0}^{T} S^{t}$. Given an initial global state $s_{0}$, the expected social welfare defined in (32) is a deterministic function of the following $\left(X \sum_{t=0}^{T} S^{t}\right)$-dimensional action vector:

$$
\left\{\nu_{t}\left(x, h_{t}\right)\right\}_{x \in \mathcal{X}_{0}, h_{t} \in \mathcal{H}\left(s_{0}\right)},
$$

where $\mathcal{H}\left(s_{0}\right)$ is the set of positive probability histories commencing at state $s_{0}$. Under Assumption 4, the expected social welfare defined in (32) is a concave function of the vector in (55). Therefore, the following conditions are necessary and sufficient for the action vector (in the form of (55)) 
associated with the DOE strategy $\nu$ to maximize the expected social welfare, among all possible dynamic oblivious strategies ${ }^{13}$ :

$$
\left\{\begin{array}{c}
\frac{\partial_{+} U_{t}\left(l_{\nu, h_{t}}\left(x_{i, 0}\right), s_{t}, \nu_{t}\left(x_{i, 0}, h_{t}\right)\right)}{\partial_{+} \nu_{t}\left(x_{i, 0}, h_{t}\right)} \leq \widetilde{p}_{t \mid \nu, h_{t}}+\widetilde{w}_{t \mid \nu, h_{t}}+1_{\tau<T} \cdot g_{t \mid \nu, h_{t}}^{+}\left(x_{i, 0}\right), \\
\quad \text { if } \nu_{t}\left(x_{i, 0}, h_{t}\right)<B \\
\frac{\partial_{-} U_{t}\left(l_{\nu, h_{t}}\left(x_{i, 0}\right), s_{t}, \nu_{t}\left(x_{i, 0}, h_{t}\right)\right)}{\partial_{-} \nu_{t}\left(x_{i, 0}, h_{t}\right)} \geq \widetilde{p}_{t \mid \nu, h_{t}}+\widetilde{w}_{t \mid \nu, h_{t}}+1_{\tau<T} \cdot g_{t \mid \nu, h_{t}}^{-}\left(x_{i, 0}\right), \\
\quad \text { if } \nu_{t}\left(x_{i, 0}, h_{t}\right)>0
\end{array}\right.
$$

where $l_{\nu, h_{t}}\left(x_{i, 0}\right)$ is consumer $i$ 's state, $x_{i, t}$, under a (positive probability) history $h_{t}$ and the strategy $\nu$ (cf. p.15), the prices, $\widetilde{p}_{t \mid \nu, h_{t}}$ and $\widetilde{w}_{t \mid \nu, h_{t}}$ are given in (17) and (18), and where, if $k_{h_{\tau}}(\cdot)$ (cf. the definition in (35)) is nondecreasing in $a_{i, t}$ for any $t<\tau \leq T$, then $g_{t \mid \nu, h_{t}}^{+}\left(x_{i, 0}\right)$ is given by ${ }^{14}$

$$
g_{t \mid \nu, h_{t}}^{+}\left(x_{i, 0}\right)=\mathbb{E}\left\{\widetilde{q}_{t+1 \mid \nu, h_{t+1}}-\sum_{\tau=t+1}^{T} \frac{\partial_{+} U_{\tau}\left(x_{i, 0}, z_{i, \tau}, s_{\tau}, a_{i, \tau}\right)}{\partial_{+} z_{i, \tau}} \cdot \frac{\partial_{+} z_{i, \tau}}{\partial_{+} a_{i, t}}\right\}, \quad \forall x_{i, 0} \in \mathcal{X}_{0},
$$

where the price, $\widetilde{q}_{t+1 \mid \nu, h_{t+1}}$, is defined in (18), the expectation is over the future global states, $\left\{s_{\tau}\right\}_{t+1}^{T}, z_{i, \tau}=k_{h_{\tau}}\left(x_{i, 0}, a_{i, 0}, \ldots, a_{i, \tau-1}\right)$ for $\tau>t$, and $a_{i, \tau}=\nu_{\tau}\left(x_{i, \tau}, h_{\tau}\right)$ for $\tau \geq t$. The expression (57) is the part of the right derivative of the expected social welfare (32) with respect to the action $a_{i, t}$, which reflects the influence of consumer $i$ 's action at stage $t$ on the ancillary cost $\widetilde{H}\left(\widetilde{A}_{t}, \widetilde{A}_{t+1}, \bar{s}_{t+1}\right)$ at the next stage, and on her future utility (due to the influence of the action $a_{i, t}$ on the future state $z_{i, \tau}$, through the functions $\left.k_{h_{\tau}}(\cdot)\right)$. In $(56), g_{t \mid \nu, h_{t}}^{-}\left(x_{i, 0}\right)$ can be defined by replacing the right (left) partial derivatives in (57) with left (respectively, right) partial derivatives.

Given an initial global state $s_{0}$, and the initial state of consumer $i, x_{i, 0}$, her oblivious value, defined in (21), is a deterministic, concave function of the vector

$$
\left\{\nu_{t}\left(x_{i, 0}, h_{t}\right)\right\}_{h_{t} \in \mathcal{H}\left(s_{0}\right)}
$$

of actions that she would take at any given stage and for any given history. Since the DOE strategy $\nu$ maximizes consumer $i$ 's oblivious value, it is easily checked that the vector in (58) must satisfy the conditions (56). Since this is true for any $x_{i, 0} \in \mathcal{X}_{0}$, we conclude that the action vector (55)

\footnotetext{
${ }^{13}$ We use the notations $\partial_{+} f$ and $\partial_{-} f$ to denote the right and left, respectively, derivatives of a function $f$.

${ }^{14}$ If for some $\tau>t, k_{h_{\tau}}(\cdot)$ is decreasing in $a_{i, t}$, then the right partial derivative of $U_{\tau}\left(x_{i, \tau}, s_{\tau}, a_{i, \tau}\right)$ with respect to $z_{i, \tau}$ in (57) should be replaced by its left partial derivative.
} 
(which is comprised by putting together the vectors in (58), for different types in the set $\mathcal{X}_{0}$ ) satisfies the conditions (56). Thus, the DOE $\nu$ satisfies the sufficient condition for optimality and the result (54) follows.

\section{D.2. Proof of Part (b)}

For a given initial global state $s_{0}$, let us fix some initial distribution $f_{0}^{n}$ with $d\left(f_{0}^{n}, \eta_{s_{0}}\right) \leq \delta$, where $\delta$ is small. In Step 1, we compare the social welfare achieved by various strategy profiles and show that

$$
\frac{1}{n} \mathcal{W}_{0}^{n}\left(f_{0}^{n}, s_{0} \mid \boldsymbol{\kappa}^{n}\right) \leq \frac{1}{n} \mathcal{W}_{0}^{n}\left(s_{0} \mid \boldsymbol{\vartheta}^{n, f_{0}^{n}}\right) \approx \widetilde{\mathcal{W}}_{0}\left(s_{0} \mid \vartheta^{n, f_{0}^{n}}\right)
$$

Here, $\kappa^{n}$ is a general history-dependent strategy profile for the $n$-consumer model (cf. (22)). The symmetric strategy profile $\boldsymbol{\vartheta}^{n, f_{0}^{n}}=\left(\vartheta^{n, f_{0}^{n}}, \ldots, \vartheta^{n, f_{0}^{n}}\right)$ is one that maximizes expected social welfare given the initial population state $f_{0}^{n}$. In Step 1, we will argue that $\vartheta^{n, f_{0}^{n}}$ can be identified with a dynamic oblivious strategy. In the approximate equality we are comparing the expected (over future global states, $\left\{s_{t}\right\}_{t=1}^{T}$ ) social welfare under the same oblivious strategy $\vartheta^{n, f_{0}^{n}}$ (hence the same sequence of actions for each consumer type $x \in \mathcal{X}_{0}$ ) under two different initial population states (initial distributions of consumer types), $f_{0}^{n}$ and $\eta_{s_{0}}$.

Since $\nu$ is a DOE, part (a) of the theorem implies that

$$
\widetilde{\mathcal{W}}_{0}\left(s_{0} \mid \vartheta^{n, f_{0}^{n}}\right) \leq \widetilde{\mathcal{W}}_{0}\left(s_{0} \mid \nu\right)
$$

Note that as the number of consumer grows large, with high probability the initial population state $f_{0}^{n}$ is close to its expectation, $\eta_{s_{0}}$. In Step 2, we complete the proof of part (b) by showing that

$$
\widetilde{\mathcal{W}}_{0}\left(s_{0} \mid \nu\right) \approx \frac{1}{n} \mathbb{E}\left\{\mathcal{W}_{0}^{n}\left(f_{0}^{n}, s_{0} \mid \boldsymbol{\nu}^{n}\right)\right\}
$$

where the expectation is over the initial population state $f_{0}^{n}$.

Step 1: If the initial population state is close to its expectation, the optimal social welfare in an $n$-consumer model can be approximated by the social welfare achieved by a dynamic oblivious strategy in the corresponding continuum model. 
In this step, we aim to show that in an $n$-consumer model, for any given initial global state $s_{0}$ and any $\varepsilon>0$, there exists some $\delta>0$ such that for any initial distribution $f_{0}^{n}$ with $d\left(f_{0}^{n}, \eta_{s_{0}}\right) \leq \delta$, we can find a dynamic oblivious strategy $\vartheta^{n, f_{0}^{n}}$ that satisfies

$$
\mathcal{W}_{0}^{n}\left(f_{0}^{n}, s_{0} \mid \boldsymbol{\kappa}^{n}\right) \leq n \widetilde{\mathcal{W}}_{0}\left(s_{0} \mid \vartheta^{n, f_{0}^{n}}\right)+\varepsilon n
$$

for all symmetric history-dependent strategy profiles, $\boldsymbol{\kappa}^{n}=\left(\kappa^{n}, \ldots, \kappa^{n}\right)$. Given an initial global state $s_{0}$ and an initial population state $f_{0}^{n}$, we observe that the social welfare, $\mathcal{W}_{0}^{n}\left(f_{0}^{n}, s_{0} \mid \boldsymbol{\kappa}^{n}\right)$, is a deterministic, concave function of the following vector of consumers' actions under different histories,

$$
\left\{\kappa_{t}^{n}\left(m_{i, \kappa^{n}, h_{t}}\left(x_{i, 0}\right), h_{t}, f_{-i, t}^{n}\right)\right\}_{h_{t} \in \mathcal{H}\left(s_{0}\right), x_{i, 0} \in \mathcal{X}_{0}, i=1, \ldots, n}
$$

where $m_{i, \kappa^{n}, h_{t}}: \mathcal{X}_{0} \rightarrow \mathcal{Y}_{t}$ maps consumer $i$ 's initial state into her augmented state at the history $h_{t}$, under the strategy profile $\boldsymbol{\kappa}^{n}$, and $f_{-i, t}^{n}$ is the distribution of other consumers' augmented states at the history $h_{t}$, under the strategy profile $\boldsymbol{\kappa}^{n}$. Note that given the initial population state $f_{0}^{n}$, the strategy profile $\boldsymbol{\kappa}^{n}$, and a history $h_{t}$, the augmented state of consumer $i$ at stage $t$ depends only on her initial state $x_{i, 0}$.

Since the social welfare $\mathcal{W}_{0}^{n}\left(f_{0}^{n}, s_{0} \mid \boldsymbol{\kappa}^{n}\right)$ is concave in the action vector in $(60)$, there exists a symmetric solution, $\vartheta^{n, f_{0}^{n}}=\left\{\vartheta_{t}^{n, f_{0}^{n}}\right\}_{t=0}^{T}$, such that if at any history $h_{t} \in \mathcal{H}\left(s_{0}\right)$, all consumers with the same initial state take the same action according to

$$
a_{i, t}=\vartheta_{t}^{n, f_{0}^{n}}\left(x_{i, 0}, h_{t}\right), \quad i=1, \ldots, n
$$

then the expected social welfare, $\mathcal{W}_{0}^{n}\left(f_{0}^{n}, s_{0} \mid \boldsymbol{\kappa}^{n}\right)$, is maximized among all possible symmetric history-dependent strategy profiles ${ }^{15}$. In (61) we have defined a dynamic oblivious strategy $\vartheta^{n, f_{0}^{n}}$ that maximizes the expected social welfare in the $n$-consumer model, conditional on the initial global state being $s_{0}$, and the initial population state being $f_{0}^{n}$. That is, in an $n$-consumer model, for any given $s_{0}$ and $f_{0}^{n}$, there exists a dynamic oblivious strategy $\vartheta^{n, f_{0}^{n}}$ such that

$$
\sup _{\kappa^{n}} \mathcal{W}_{0}^{n}\left(f_{0}^{n}, s_{0} \mid \boldsymbol{\kappa}^{n}\right)=\mathcal{W}_{0}^{n}\left(f_{0}^{n}, s_{0} \mid \boldsymbol{\vartheta}^{n, f_{0}^{n}}\right),
$$

\footnotetext{
15 The fact that the supremum is attained is a consequence of our continuity assumption and the fact that the various variables of interest can be restricted to be in a compact set.
} 
where $\boldsymbol{\vartheta}^{n, f_{0}^{n}}=\left(\vartheta^{n, f_{0}^{n}}, \ldots, \vartheta^{n, f_{0}^{n}}\right)$ is the corresponding symmetric dynamic oblivious strategy profile. To verify (59), it suffices to show that for any $\varepsilon>0$, there exists some $\delta>0$ such that for any $f_{0}^{n}$ with $d\left(f_{0}^{n}, \eta_{s_{0}}\right) \leq \delta$,

$$
\left|\mathcal{W}_{0}^{n}\left(f_{0}^{n}, s_{0} \mid \boldsymbol{\vartheta}^{n, f_{0}^{n}}\right)-n \widetilde{\mathcal{W}}_{0}\left(s_{0} \mid \vartheta^{n, f_{0}^{n}}\right)\right| \leq \varepsilon n,
$$

i.e., if all consumers use the strategy $\vartheta^{n, f_{0}^{n}}$, the difference between the optimal social welfare achieved in an $n$-consumer model and the social welfare achieved in the corresponding continuum model can be made arbitrarily small, if the initial population state is close enough to its expectation, $\eta_{s_{0}}$. We next argue that the result in (63) holds for any dynamic oblivious strategy $\vartheta$.

To prove (63), we first upper bound the difference between the supplier cost in an $n$-consumer model and that in the corresponding continuum model. Since all cost functions are Lipschitz continuous (see Eqs. (27) and (28)), for any $\varepsilon>0$, there exists some $\delta_{1}>0$ such that if

$$
\left|A_{t}^{n}-n \widetilde{A}_{t \mid \vartheta, h_{t}}\right| \leq X \delta_{1} B n, \quad t=0, \ldots, T, \quad \forall h_{t} \in \mathcal{H}\left(s_{0}\right),
$$

then

$$
\begin{gathered}
\left|C^{n}\left(A_{t}^{n}, s_{t}\right)-C^{n}\left(n \widetilde{A}_{t \mid \vartheta, h_{t}}, s_{t}\right)\right| \leq n \varepsilon /(3 T+3), \quad t=0, \ldots, T, \quad \forall h_{t} \in \mathcal{H}_{t}\left(s_{0}\right), \\
\left|H_{0}^{n}\left(A_{0}^{n}, s_{0}\right)-H_{0}^{n}\left(n \widetilde{A}_{0 \mid \vartheta, h_{0}}, s_{0}\right)\right| \leq n \varepsilon /(3 T+3),
\end{gathered}
$$

and for $t=1, \ldots, T$,

$$
\left|H^{n}\left(A_{t-1}^{n}, A_{t}^{n}, \bar{s}_{t}\right)-H^{n}\left(n \widetilde{A}_{t-1 \mid \vartheta, h_{t-1}}, n \widetilde{A}_{t \mid \vartheta, h_{t}}, \bar{s}_{t}\right)\right| \leq n \varepsilon /(3 T+3), \quad \forall h_{t} \in \mathcal{H}_{t}\left(s_{0}\right)
$$

where $\mathcal{H}_{t}\left(s_{0}\right)$ is the set of all histories of length $t+1$ commencing at state $s_{0}$. Given an initial population state $f_{0}^{n}$, if all consumers use the strategy $\vartheta$, the aggregate demand under a history $h_{t}$ is

$$
A_{t}^{n}=n \sum_{x \in \mathcal{X}_{0}} f_{0}^{n}(x) \vartheta_{t}\left(x, h_{t}\right)
$$

From (16) we observe that if $d\left(f_{0}^{n}, \eta_{s_{0}}\right) \leq \delta_{1}$, the condition in (64) holds, and then Eqs. (65)-(67) are verified. 
We now show that if the initial population state is close to its expectation, the total utility obtained by all consumers is close to its counterpart in the corresponding continuum model. Given an initial population state $f_{0}^{n}$, we write the total utility obtained by all consumers under a history $h_{t}$ as

$$
\sum_{i=1}^{n} U_{t}\left(x_{i, t}, s_{t}, a_{i, t}\right)=n \sum_{x \in \mathcal{X}_{0}} f_{0}^{n}(x) U_{t}\left(l_{\vartheta, h_{t}}(x), \vartheta_{t}\left(x, h_{t}\right), s_{t}\right) .
$$

On the other hand, the utility achieved in the corresponding continuum model is given by

$$
\widetilde{U}_{t \mid \vartheta, h_{t}} \triangleq \sum_{x \in \mathcal{X}_{0}} \eta_{s_{0}}(x) U_{t}\left(l_{\vartheta, h_{t}}(x), \vartheta_{t}\left(x, h_{t}\right), s_{t}\right)
$$

We have that if $d\left(f_{0}^{n}, \eta_{s_{0}}\right) \leq \varepsilon /(3 X Q(T+1))$, then

$$
\left|\sum_{i=1}^{n} U_{t}\left(x_{i, t}, s_{t}, a_{i, t}\right)-n \widetilde{U}_{t \mid \vartheta, h_{t}}\right| \leq n \varepsilon /(3 T+3), \quad t=0, \ldots, T, \quad \forall h_{t} \in \mathcal{H}_{t}\left(s_{0}\right), \quad \forall n \in \mathbb{N}^{+},
$$

Let $\delta=\min \left\{\delta_{1}, \varepsilon /(3 X Q(T+1))\right\}$. If $d\left(f_{0}^{n}, \eta_{s_{0}}\right) \leq \delta$, from $(65)-(68)$ we have

$$
\left|\widetilde{W}_{t}\left(h_{t} \mid \vartheta\right)-W_{t}^{n}\left(f_{t}^{n}, h_{t} \mid \vartheta^{n}\right)\right| \leq n \varepsilon /(T+1), \quad t=0, \ldots, T, \quad \forall h_{t} \in \mathcal{H}_{t}\left(s_{0}\right)
$$

Eq. (63) follows from the definition of expected social welfare in an $n$-consumer model (31), and in a continuum model (32). The desired result in (59) follows.

\section{Step 2: Asymptotic social optimality of a DOE.}

In this step, we complete the proof of part (b) of the theorem, using the fact that as the number of consumers grows large, with high probability the initial population state is close to its expectation. Note that the action space is $[0, B]$, so that $\left|A_{t}\right| \leq n B$. Using Assumption $3, C^{n}(\cdot) / n$ is therefore bounded. A similar argument holds for $H_{0}^{n}(\cdot) / n$ and $H^{n}(\cdot) / n$. Furthermore, the total utility per consumer is also bounded. Thus, there exists some constant $D$ that upper bounds $\left|\mathcal{W}_{0}^{n} / n\right|$. We define $\mathfrak{F}_{s_{0}}^{n}(\delta)$ as the set of initial population states such that $d\left(f_{0}^{n}, \eta_{s_{0}}\right) \leq \delta$. By the law of large numbers, for any pair of positive real numbers, $\varepsilon$ and $\delta$, we can find an integer $N$ such that

$$
\sum_{f_{0}^{n} \notin \mathfrak{F}_{s_{0}}^{n}(\delta)} \mathbb{P}\left(F_{s_{0}}^{n}=f_{0}^{n}\right) \cdot \sup _{\kappa^{n}}\left|\mathcal{W}_{0}^{n}\left(f_{0}^{n}, s_{0} \mid \boldsymbol{\kappa}^{n}\right)\right| \leq D \mathbb{P}\left(d\left(f_{0}^{n}, \eta_{s_{0}}\right)>\delta\right) \leq \varepsilon n, \quad \forall n \geq N
$$


For any $\varepsilon>0$, let $\delta$ be the positive real number defined in (59), and let $N$ be the positive integer given in (69); for any $n \geq N$ and any symmetric history-dependent strategy profile $\boldsymbol{\kappa}^{n}$, we have

$$
\begin{aligned}
& \mathbb{E}\left\{\mathcal{W}_{0}^{n}\left(f_{0}^{n}, s_{0} \mid \boldsymbol{\kappa}^{n}\right)\right\} \\
\leq & \sum_{f_{0}^{n} \in \mathfrak{F}_{s_{0}}^{n}(\delta)} \mathbb{P}\left(F_{s_{0}}^{n}=f_{0}^{n}\right) \cdot \mathcal{W}_{0}^{n}\left(f_{0}^{n}, s_{0} \mid \boldsymbol{\kappa}^{n}\right)+\varepsilon n \\
\leq & \sum_{f_{0}^{n} \in \mathfrak{F}_{s_{0}}^{n}(\delta)} \mathbb{P}\left(F_{s_{0}}^{n}=f_{0}^{n}\right) \cdot\left(n \widetilde{\mathcal{W}}_{0}\left(s_{0} \mid \vartheta^{n, f_{0}^{n}}\right)+\varepsilon n\right)+\varepsilon n \\
\leq & \sum_{f_{0}^{n} \in \mathfrak{F}_{s_{0}}^{n}(\delta)} \mathbb{P}\left(F_{s_{0}}^{n}=f_{0}^{n}\right) \cdot\left(n \widetilde{\mathcal{W}}_{0}\left(s_{0} \mid \nu\right)+\varepsilon n\right)+\varepsilon n \\
\leq & \sum_{f_{0}^{n} \in \mathfrak{F}_{s_{0}}^{n}(\delta)} \mathbb{P}\left(F_{s_{0}}^{n}=f_{0}^{n}\right) \cdot\left(\mathcal{W}_{0}^{n}\left(f_{0}^{n}, s_{0} \mid \boldsymbol{\nu}^{n}\right)+2 \varepsilon n\right)+\varepsilon n \\
\leq & \mathbb{E}\left\{\mathcal{W}_{0}^{n}\left(f_{0}^{n}, s_{0} \mid \boldsymbol{\nu}^{n}\right)\right\}+4 \varepsilon n,
\end{aligned}
$$

where the first inequality follows from (69), the second inequality is due to (59), the third inequality follows from the optimality property of the DOE $\nu$ (part (a) of the theorem), the fourth inequality follows similar to (63) (the proof of Eq. (63) remains valid for any dynamic oblivious strategy), and the last inequality follows from (69).

\section{E. Numerical Results}

In this section we give a numerical example to compare the proposed pricing mechanism with marginal cost pricing. The comparison is carried out in terms of DOEs and the resulting social welfare under the corresponding continuum model. Towards this purpose, we first define the DOE for a continuum model under the marginal cost pricing mechanism, in Section E.1. In Section E.2, we consider a two-stage dynamic model in which the consumers' marginal utility and demand increase at the second stage. We calculate the equilibria resulting from the two pricing mechanisms, and compare the potential of the two pricing mechanisms to improve social welfare and reduce peak load.

\section{E.1. Equilibrium under Marginal Cost Pricing}

In an $n$-consumer model, at stage $t \geq 1$, the supplier's marginal cost is

$$
\left(C^{n}\right)^{\prime}\left(A_{t}^{n}, s_{t}\right)+\frac{\partial H^{n}\left(A_{t-1}^{n}, A_{t}^{n}, \bar{s}_{t}\right)}{\partial A_{t}^{n}}=p_{t}^{n}+w_{t}^{n}, \quad t=1, \ldots, T .
$$

At stage 0, the supplier's marginal cost is

$$
\left(C^{n}\right)^{\prime}\left(A_{0}^{n}, s_{0}\right)+\left(H_{0}^{n}\right)^{\prime}\left(A_{0}^{n}, s_{0}\right)=p_{0}^{n}+w_{0}^{n} .
$$


Under marginal cost pricing, each consumer's stage payoff is

$$
\pi\left(y_{i, t}, \bar{s}_{t}, a_{i, t}, f_{-i, t}^{n}, u_{-i, t}^{n}\right)=U\left(x_{i, t}, s_{t}, a_{i, t}\right)-\left(p_{t}^{n}+w_{t}^{n}\right) \cdot a_{i, t},
$$

where the stage marginal cost, $p_{t}^{n}+w_{t}^{n}$, is given in (70) and (71), and $y_{i, t}=\left(x_{i, t}, a_{i, t-1}\right)$.

For marginal cost pricing, we now define the nonatomic equilibrium concept in the corresponding continuum model. Suppose that all consumers other than $i$ use a dynamic oblivious strategy $\nu$. Consumer $i$ 's oblivious stage value under marginal cost pricing is given by

$$
\tilde{\pi}_{i, t}\left(y_{i, t}, \bar{s}_{t}, f_{t \mid \nu, h_{t}}, a_{i, t} \mid \nu\right)=U_{t}\left(x_{i, t}, s_{t}, a_{i, t}\right)-\left(\widetilde{p}_{t \mid \nu, h_{t}}+\widetilde{w}_{t \mid \nu, h_{t}}\right) \cdot a_{i, t},
$$

where $\widetilde{p}_{t \mid \nu, h_{t}}$ and $\widetilde{w}_{t \mid \nu, h_{t}}$ are defined in (17) and (18). Replacing the oblivious stage value function in (19) with that given in (73), we can define an equilibrium concept for the marginal cost pricing mechanism in a similar way as for the DOE in Section 4.

\section{E.2. Numerical Example}

In current wholesale electricity markets, we observe that the highest daily wholesale price usually occurs when the system load increases quickly (cf. Fig. 1 in Section 1). Inspired by the above observation, we construct a two-stage dynamic model, in which the aggregate demand increases quickly at the second stage, to compare the performance of the proposed mechanism with marginal cost pricing. For simplicity, we assume that there is a continuum of identical consumers indexed by $i \in[0,1]$. Each consumer would like to consume $1+x$ and $1.2-x$ at the two stages, where $x \in[0, E]$. Here, $E \in[0,0.1]$ (a given constant) is the amount of electricity demand that can be shifted from the second stage to the first stage. The value of $E$ will be called demand substitutability ${ }^{16}$.

Formally, consumer $i$ 's state at each stage denotes the maximum amount of electricity she could use at the stage ${ }^{17}$. For a given consumer $i$, we have $x_{i, 0}=1+E$, and her state at stage 1 is determined as follows:

\footnotetext{
16 There are two types of elasticity of consumers' demand: (i) consumers may curtail their demand at a high price, and (ii) they may shift their demand to a less expensive time. The first type of demand response is a price elasticity, and the second type is an elasticity of substitution across time. The first type of elasticity is incorporated in our model through the utility functions, and the second type of elasticity is incorporated through $E$.

${ }^{17}$ Since all consumers are of the same type, the consumer state space in this example is a subset of $[0, \infty)$.
} 
1. if $a_{i, 0} \leq 1$, the maximum amount of electricity she could use at stage 1 is $1.2-E+E$, i.e., $x_{i, 1}=1.2$

2. if $1<a_{i, 0} \leq x_{i, 0}$, the maximum amount of electricity she could use at stage 1 is $x_{i, 1}=1.2-$ $E+\left(x_{i, 0}-a_{i, 0}\right)=2.2-a_{i, 0}$

3. if $x_{i, 0}<a_{i, 0}$, the maximum amount of electricity she could use at stage 1 is $x_{i, 1}=1.2-E$.

To summarize, we have

$$
x_{i, 1}=1.2-E+\max \left\{0, x_{i, 0}-\max \left\{a_{i, 0}, 1\right\}\right\} .
$$

For each stage $t$, the utility functions are given by

$$
U_{t}\left(x_{i, t}, s_{t}, a_{i, t}\right)= \begin{cases}d_{t} a_{i, t}, & \text { if } 0 \leq a_{i, t} \leq x_{i, t}, \\ d_{t} x_{i, t}, & \text { if } a_{i, t}>x_{i, t}\end{cases}
$$

where the slopes are $d_{0}=10$ and $d_{1}=12$. Here, we assumed that the consumers place a larger value on electricity during peak hours, and that shifting peak load to off-peak hours hurts consumer utility. For example, rescheduling kitchen and laundry activities may cause inconvenience for residential consumers; similarly, industrial consumers may face higher labor cost premiums for off-peak production.

The primary cost function (cf. Section 2) is $\widetilde{C}(A, s)=A^{2}$, for any $s$. We assume that the capacity available at each stage is proportional to the system load, i.e.,

$$
G_{t}=b_{t} A_{t}, \quad t=0,1
$$

and that the ancillary cost depends only on the difference between the capacity available at two consecutive stages. At the second stage (peak hour), we assume that the system operator maintains a reserve margin of $10 \%$, i.e., $b_{1}=1.1$. We will consider two different system operator policies: (i) the system operator does not forecast the load jump at the second stage, and uses a conservative policy under which $b_{0}=1.12$, and (ii) the system operator predicts the load jump at the second stage, and ramps up the system capacity in advance, by letting $b_{0}=1.2$. 
For simplicity, we use a quadratic function to approximate the ancillary cost associated with load fluctuations:

$$
\widetilde{H}_{0}\left(A_{0}, s_{0}\right)=10\left(\max \left\{b_{0} A_{0}-1.12,0\right\}\right)^{2}, \quad \widetilde{H}\left(A_{0}, A_{1}, \bar{s}_{1}\right)=20\left(\max \left\{b_{1} A_{1}-b_{0} A_{0}, 0\right\}\right)^{2},
$$

where 1.12 represents the capacity available at the stage before the initial stage ${ }^{18}$. We assumed a higher coefficient, 20, for the ancillary cost at the second stage, due to the increase of the system load.

For different levels of demand substitutability $E$, and two different system operator policies $\left(b_{0}\right.$ equal to 1.12 or $b_{0}=1.2$ ), we compare the social welfare (in Section E.2.1) and the peak load (in Section E.2.2) resulting from the equilibria of the two pricing mechanisms.

E.2.1. Social welfare gain. For various levels of demand substitutability $(E \in[0,0.1])$, and the two different system operator policies, we calculate the equilibria resulting from the two pricing mechanisms. Fig. 3 compares the social welfare achieved by the proposed mechanism and the marginal cost pricing mechanism. We observe from Fig. 3 the following.

1. System operator's policy: When the consumers have a low level of demand substitutability, the policy with $b_{0}=1.2$ achieves a much higher social welfare than the conservative policy $\left(b_{0}=\right.$ 1.12), under both the proposed and the marginal cost pricing mechanisms. (This is to be expected, because when $b_{0}=1.12$, and with the demand at stage 1 more or less fixed, the difference $b_{1} A_{1}-b_{0} A_{0}$ is necessarily large.) For consumers with a high level of demand substitutability, the policy with $b_{0}=1.2$ achieves a slightly smaller social welfare than the conservative policy $\left(b_{0}=1.12\right)$, because the policy with $b_{0}=1.2$ results in a lower price at the second stage than the conservative one, and therefore does not provide enough encouragement to the consumers to shift their peak load (cf. the discussion in Section E.2.2).

2. Social welfare gain at a low level of demand substitutability: At a low level of demand substitutability, e.g., when $E \leq 0.02$, and under the system operator's conservative policy

\footnotetext{
${ }^{18}$ Suppose that the load at stage " -1 " is 1 , and that the capacity available at stage -1 is 1.12 , under an average
} reserve margin of $12 \%$. 


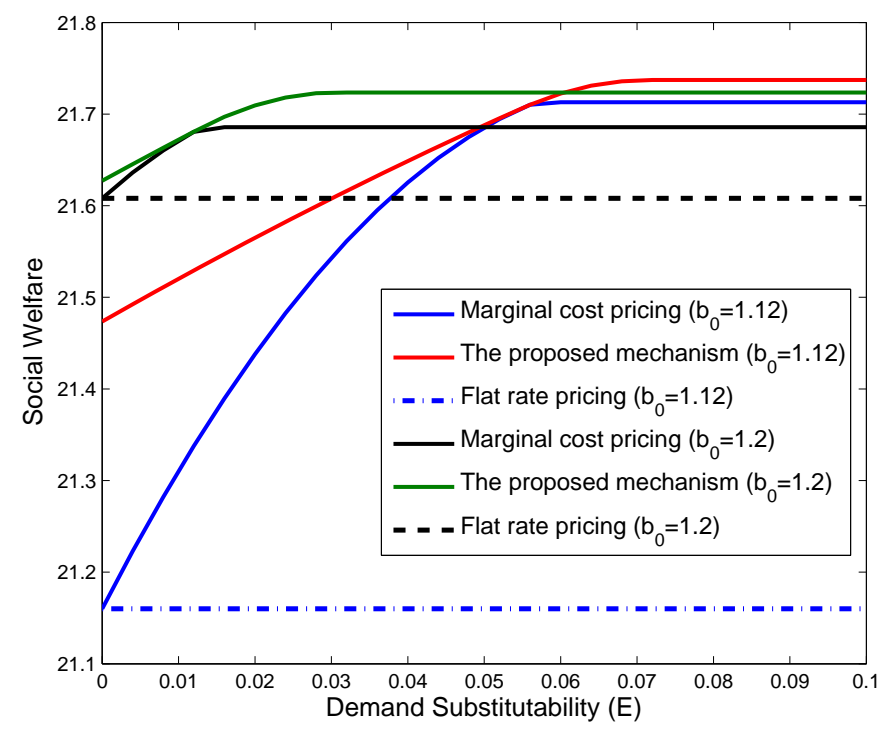

Figure 3 The social welfare achieved by the proposed pricing mechanism, the marginal cost pricing mechanism and the flat rate pricing mechanism, as a function of the demand substitutability, $E$.

$\left(b_{0}=1.12\right)$, we observe that the proposed pricing mechanism achieves significantly more social welfare gain (the social welfare achieved by flat rate pricing ${ }^{19}$ is used a reference) than marginal cost pricing; if the system operator ramps up the capacity in advance $\left(b_{0}=1.2\right)$, both pricing mechanisms achieve approximately the same social welfare as flat rate pricing.

3. Social welfare gain at a high level of demand substitutability: If the consumers have a high demand substitutability, e.g., when $E \geq 0.08$, the proposed pricing mechanism achieves approximately $5 \%$ more social welfare gain than marginal cost pricing under the system operator's conservative policy $\left(b_{0}=1.12\right)$; if the system operator ramps up the capacity in advance $\left(b_{0}=\right.$ 1.2), the proposed pricing mechanism achieves approximately $50 \%$ more social welfare gain than marginal cost pricing.

Let us now derive some insights by considering the special case of zero demand substitutability $(E=0)$ and $b_{0}=1.12$. The one-stage aggregate demand and the social welfare resulting from the

${ }^{19}$ Under flat rate pricing, consumers pay a fixed (time-invariant) retail price for the electricity they consume. Since the average retail price is less than the consumers' marginal utility (see Tables 2 and 4), the payoff-maximizing consumer demand at the two stages is 1 and 1.2, respectively. Since all consumers are identical, the aggregate demand at the two stages is 1 and 1.2 . 
Table 1 Demand and social welfare (per consumer) at $E=0$ and $b_{0}=1.12$

\begin{tabular}{cccc}
\hline \hline & $a_{0}$ & $a_{1}$ & Social welfare \\
\hline Flat rate & 1 & 1.2 & 21.16 \\
Marginal cost & 1 & 1.2 & 21.16 \\
Proposed & 1.0901 & 1.2 & 21.4735
\end{tabular}

Table 2 Price fluctuation at $E=0$ and $b_{0}=1.12$. The price $p_{t}+w_{t}$ equals the marginal cost at stage $t$.

\begin{tabular}{ccccc}
\hline \hline & $p_{0}+w_{0}$ & $p_{1}+w_{1}$ & $q_{1}$ & Average (retail) price \\
\hline Flat rate & 2 & 11.2 & - & 7.0182 \\
Marginal cost & 2 & 11.2 & - & 7.0182 \\
Proposed & 4.4401 & 6.7609 & -4.4401 & 5.6562
\end{tabular}

three pricing mechanisms are given in Table 1. The prices faced by consumers are given in Table 2 , where the average retail price is the ratio of the total money a consumer pays at an equilibrium to her total demand during the two stages. ${ }^{20}$ Note that under the proposed pricing mechanism, a consumer pays

$$
\left(p_{0}+w_{0}+q_{1}\right) a_{i, 0}+\left(p_{1}+w_{1}\right) a_{i, 1},
$$

while she would pay $\left(p_{0}+w_{0}\right) a_{i, 0}+\left(p_{1}+w_{1}\right) a_{i, 1}$ under marginal cost pricing. In general, the price $q_{1}$ will be negative and will be even smaller if we were to increase the aggregate demand at the second stage. That is, a higher peak load results in a lower price at the first stage, which encourages consumers to increase their demand at the off-peak hour, even if they do not derive any additional utility from such an increase. In fact, from Table 2 we observe that at the DOE, the proposed pricing mechanism offers each consumer a zero total price on $a_{0}$. This may appear illogical at first sight. The reason is that due to the conservative reserve policy, with $b_{0}=1.12$, a demand of $a_{0}=1$ results in a large increase from $b_{0} a_{0}$ to $b_{1} a_{1}$ and hence a large ancillary cost. The increase of the demand $a_{0}$ beyond 1 does not provide any utility to the consumer, but reduces the ancillary cost. Thus, the counterintuitive choice of $a_{0}=1.0901$ serves to mitigate a conservative and somewhat deficient reserve policy. This suggests that further research is needed that will include an intertemporal optimization of the reserve policy as well.

\footnotetext{
${ }^{20}$ Note that only consumers under flat rate pricing pay this price. We list the average prices for the two real-time pricing mechanisms to compare the consumers' expense under different pricing mechanisms.
} 
Table 3 Demand and social welfare (per consumer) at $E=0.08$ and $b_{0}=1.2$

\begin{tabular}{cccc}
\hline \hline & $a_{0}$ & $a_{1}$ & Social welfare \\
\hline Flat rate & 1 & 1.2 & 21.608 \\
Marginal cost & 1.0131 & 1.1869 & 21.6857 \\
Proposed & 1.0308 & 1.1692 & 21.7237
\end{tabular}

Table 4 Price fluctuation at $E=0.08$ and $b_{0}=1.2$. The price $p_{t}+w_{t}$ equals the marginal cost at stage $t$.

\begin{tabular}{ccccc}
\hline \hline & $p_{0}+w_{0}$ & $p_{1}+w_{1}$ & $q_{1}$ & Average (retail) price \\
\hline Flat rate & 3.92 & 7.68 & - & 5.971 \\
Marginal cost & 4.324 & 6.324 & - & 5.403 \\
Proposed & 4.868 & 4.505 & -2.363 & 3.568
\end{tabular}

For the case where the system operator ramps up the capacity in advance $\left(b_{0}=1.2\right)$, and consumers have a high level of demand substitutability $(E=0.08)$, the one-stage aggregate demand and the social welfare resulting from the three pricing mechanisms are given in Table 3 . The prices faced by consumers are given in Table 4 . From Table 3 we observe that under the proposed pricing mechanism, consumers would like to shift 0.031 peak load to off-peak hours, while under the marginal cost pricing mechanism, consumers are willing to shift less than 0.014 peak load to offpeak hours. Compared to marginal cost pricing, the more flattened load curve resulting from the proposed pricing mechanism leads to $50 \%$ more social welfare gain.

For a given load curve, the proposed pricing mechanism results in a larger price difference between stage 1 and stage 0 than marginal cost pricing, because of the negative price $q_{1}$. The negative price $q_{1}$ creates an additional incentive for consumers to shift their load from stage 1 to stage 0 . In this way, the proposed pricing mechanism results in a more flattened load curve and a higher social welfare than marginal cost pricing (cf. Table 3).

E.2.2. Peak load reduction. Under flat rate pricing, the peak load (the aggregate demand at the second stage) is 1.2 , because consumers do not have an incentive to shift their load to off-peak hours. Given a pricing mechanism and a system operator's policy $\left(b_{0}\right)$, consumers are willing to substitute across time only up to a certain level. Even with a high level of demand substitutability, consumers prefer not to shift much of their peak load, to avoid the utility loss caused by peak load shifting. For example, with $b_{0}=1.2$ and $E=0.08$, consumers under marginal cost pricing choose 


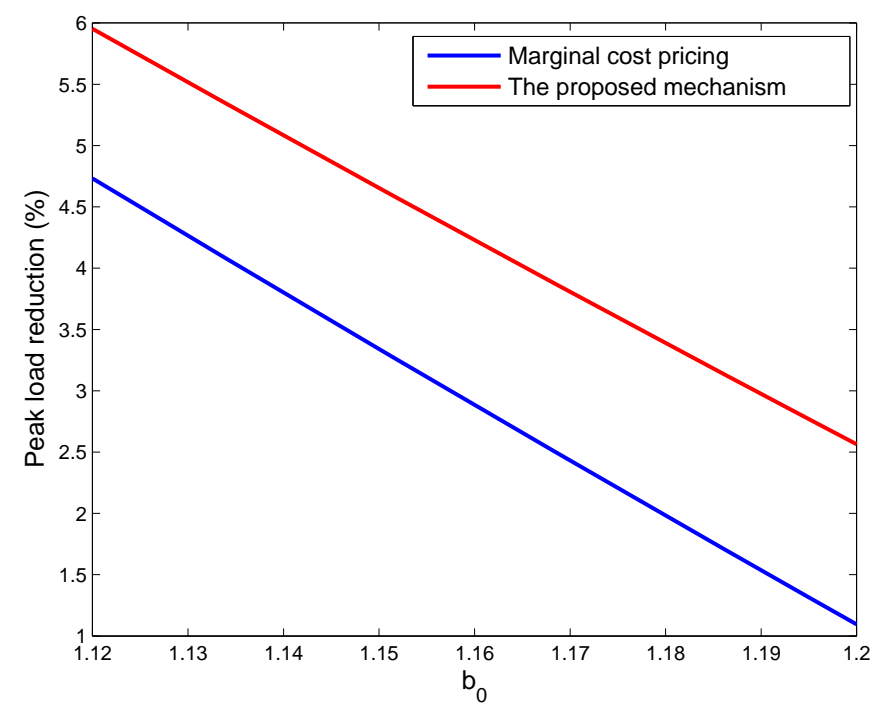

Figure 4 Comparison of the percentage of peak load reduction (the peak load under flat rate pricing, 1.2, is used a reference) resulting from the proposed pricing mechanism and the marginal cost pricing mechanism, as a function $b_{0}$.

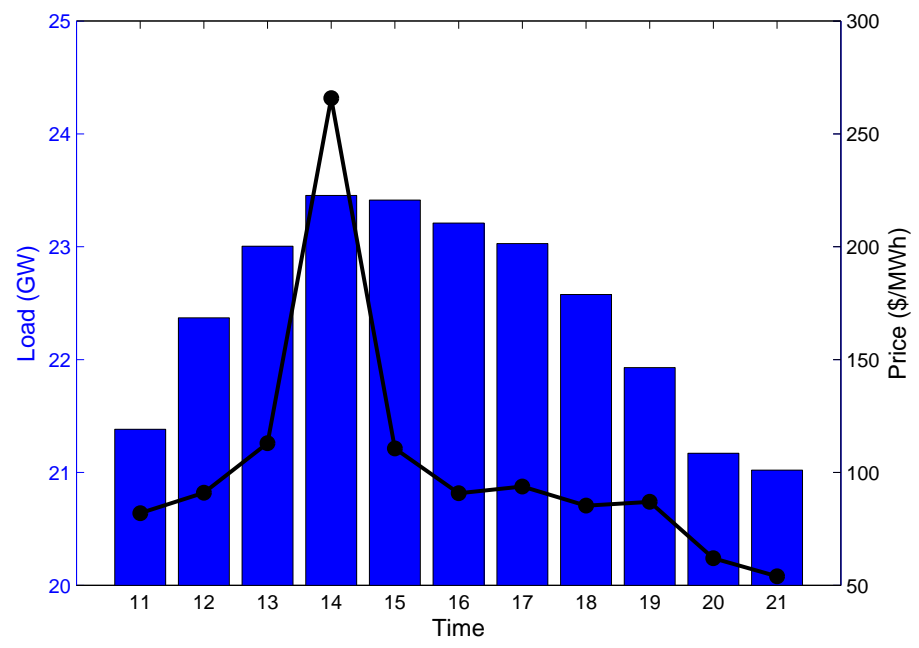

Figure 5 Real-time prices and actual system loads on August 01, 2011, ISO New England Inc. Blue bars represent the real-time system loads and the dots connected by a black line represent the hourly prices.

to shift at most 0.013 peak load (cf. Table 3). In Fig. 4, for different values of $b_{0}$, we compare the maximum amount of peak load consumers choose to shift under the proposed pricing mechanism and the marginal cost pricing mechanism.

We observe from Fig. 4 that the amount of peak load consumers will shift decreases with $b_{0}$. 
This is because a larger reserve at the first stage lowers the price at the second stage, which in turn discourages consumers from shifting their peak load. The proposed pricing mechanism results in a peak load which is approximately 1.5 percent lower than that resulting from marginal cost pricing, regardless of the value of $b_{0}$. If the system operator ramps up the system capacity in advance $\left(b_{0}=1.2\right)$, marginal cost pricing reduces the system peak load resulting from flat rate pricing by approximately one percent. Compared to marginal cost pricing, the negative price $q_{1}$ in the proposed mechanism encourages consumers to make a larger shift of their peak load (cf. the discussion at the end of Section E.2.1).

Fig. 5 plots the real-time system loads and prices on August 1, 2011, a typical hot summer day in New England ${ }^{21}$. If consumers are able to shift some of their load to the morning (possibly at the expense of losing some utility), the proposed pricing mechanism encourages consumers to shift more of their peak load than marginal cost pricing. Since the highest peak load determines the generation capacity necessary for system reliability, the proposed pricing mechanism has a greater potential to reduce the long-term capacity investment.

${ }^{21}$ www.ferc.gov/market-oversight/mkt-electric/new-england/2011/08-2011-elec-isone-dly.pdf 